\title{
Construction of Classes of Circuit-Independent Chaotic Oscillators Using Passive-Only Nonlinear Devices
}

\author{
Ahmed S. Elwakil, Member, IEEE, and Michael Peter Kennedy, Fellow, IEEE
}

\begin{abstract}
Two generic classes of chaotic oscillators comprising four different configurations are constructed. The proposed structures are based on the simplest possible abstract models of generic second-order $R C$ sinusoidal oscillators that satisfy the basic condition for oscillation and the frequency of oscillation formulas. By linking these sinusoidal oscillator engines to simple passive first-order or second-order nonlinear composites, chaos is generated and the evolution of the two-dimensional sinusoidal oscillator dynamics into a higher dimensional state space is clearly recognized. We further discuss three architectures into which autonomous chaotic oscillators can be decomposed. Based on one of these architectures we classify a large number of the available chaotic oscillators and propose a novel reconstruction of the classical Chua's circuit. The well-known Lorenz system of equations is also studied and a simplified model with equivalent dynamics, but containing no multipliers, is introduced.
\end{abstract}

Index Terms-Chaos, chaotic oscillators, Chua's circuit, Lorenz system, oscillators.

\section{INTRODUCTION}

$\mathbf{T}$ HE DESIGN of chaotic oscillators has been a subject of increasing interest during the past few years. As an active research topic, it has also advanced significantly due to the many contributions of different researchers [1]-[12]. The main thrust of this research area has been to introduce new chaotic oscillator circuits and to further concentrate on studying the nonlinear dynamics responsible for chaos generation in these circuits [13]-[15]. The achievement in most cases has been a new chaotic oscillator circuit but not a design methodology by which even more circuits can be generated. The main reason for this approach, in our opinion, is the lack of a set of necessary and sufficient mathematical conditions for chaos generation. Since early chaotic oscillators were introduced by researchers with more of a mathematical background than a circuit-design background, they might have been convinced that with the lack of such conditions, it is impossible to arrive at a completely systematic procedure for designing chaotic oscillators. However, we have chosen to deviate from this main research stream and adopt a point of view which is biased toward circuit-design.

Manuscript received December 15, 1999; revised July 27, 2000. This work was supported in part by the Enterprise Ireland Basic Research Program under Grant SC/98/740. This paper was recommended by Associate Editor C. K. Tse.

A. S. Elwakil is with the Department of Electric and Electronic Engineering, University of Sharjah, Emirates (email:elwakil@ee.ucd.ie).

M. P. Kennedy is with the Department of Microelectronic Engineering, Faculty of Engineering, University College Cork, Cork, Ireland (email: peter.kennedy@ucc.e).

Publisher Item Identifier S 1057-7122(01)01395-2.
Practical analog circuit designers know that for a design to fulfill its specifications with zero trial-and-error effort is an impossible mission. In particular, the available design and modeling techniques cannot take into consideration every aspect of the design. For this reason, CAD tools are heavily relied upon to minimize the essential trial and error effort. Nevertheless, most design techniques are considered systematic because they offer a start-to-end design cycle with a product that requires a small and identified margin of trial and error. If we can propose such a design cycle for chaotic oscillators with appropriate design rules, and identify clearly where trial and error is to be performed, then we have a systematic design process. We have proposed such a design cycle in [16] and [17].

One of the most important design rules proposed in [17] is to employ passive-only nonlinear devices. This rule remains strongly recommended so long as there is no evidence that the statistical properties of a chaotic signal produced by a chaotic oscillator with an active nonlinearity (ex. Chua's diode) possess any additional features which are not possessed by signals produced by chaotic oscillators with passive-only nonlinear devices. Passive nonlinear devices (ex. diodes) require no design effort and impose no restriction on the performance of a chaotic oscillator in terms of power dissipation, minimum supply voltage, or frequency response. Therefore, a designer can concentrate on the active parts of the chaotic oscillator, which operate only in linear mode, in order to enhance its performance or introduce features of particular interest.

To this end, the design/optimization process for chaotic oscillators relies primarily on techniques which are well-established in the linear circuit theory of design, requiring minimum knowledge of nonlinear dynamics. We emphasize here that the techniques proposed for chaotic oscillator design cannot guarantee that a chaotic signal with predefined statistical properties will be produced but they can be used to fulfill any circuit synthesis/performance requirements. The mapping of statistical properties into circuit-design properties can only be done when a unique set of statistical properties that can fully describe, identify, and compare chaotic signals has been devised. This theoretical problem has not yet been solved.

By observing that the design methodology proposed in [17] is centered around the idea of a core sinusoidal oscillator that is to be designed and later modified for chaos using a passive nonlinear composite, two major questions have to be answered. The first question asks: Is it possible that any of the chaotic oscillators (or systems) that were reported earlier is also based on a core sinusoidal oscillator engine? The second question asks: Is 
the active nonlinearity in these oscillators (systems) really necessary?

To answer these questions, we have carried out a series of investigations on some well-known chaotic systems including, Chua's circuit [1], Saito's double screw hysteresis oscillator [5], and Rössler's system [18]. As anticipated, we have found that all of these systems are built upon a core sinusoidal oscillator and that the active-type nonlinearity is not essential for generating chaos [19]-[21]. Therefore, we have conjectured the following [19].

Conjecture 1: In any analog continuous-time chaotic oscillator which is capable of exhibiting simple limit cycle behavior, there exists a core oscillator providing an unstable pair of complex conjugate eigenvalues and a control parameter which can move this pair. Accordingly, at least one chaotic oscillator can be derived from any sinusoidal oscillator. The derivation process requires a nonlinearity which is not necessarily active.

In this work, we introduce two classes of generic chaotic oscillators based on the simplest possible models for circuit-independent $R C$ sinusoidal oscillators. The sinusoidal oscillator is treated as a black box with its internal structure left to be decided according to a set of circuit-design specifications.

Following an interesting property of one of the proposed structures, whereby the switching of a single parameter produces a mirror image of the attractor without changing its dynamics, we have been able to construct a Lorenz-like butterfly attractor. This further motivates us to study closely the Lorenz system of equations [22], hoping to identify a similar mechanism. The result is a novel simplified model of the Lorenz system with no multipliers.

We also propose two extremely simple mathematical models, one of which is capable of capturing the essential dynamics of third-order chaotic oscillators with an anti-symmetrical switching nonlinearity, while the other captures the essential dynamics of third-order oscillators with an odd symmetrical nonlinearity. Both chaotic oscillator models are based on a core quadrature sinusoidal oscillator engine.

Finally, we discuss three general architectures for autonomous chaotic oscillators. Based on one of these architectures, we classify a collection of known oscillators in tabular form. Chua's circuit does not fit neatly into this classification. However, we can reconstruct the circuit to show that it is not an exception to the rest of the table entries. In fact, we demonstrate that Chua's circuit is based on the classical negative resistance sinusoidal oscillator and we introduce a reconstructed version based on a generic $R C$ oscillator.

\section{Class I ChaOtic OSCILlators}

In this section, we present a class of chaotic oscillators which is based on the general second-order sinusoidal oscillator shown in Fig. 1(a) and characterized by having a separate parallel $R_{1} C_{1}$ branch supplied by a current $I$ from a first-order active network. A large number of classical as well as new sinusoidal oscillators with this structure can be found in the literature [23]-[26]. The parallel $R_{1} C_{1}$ network is usually known as the timing network.

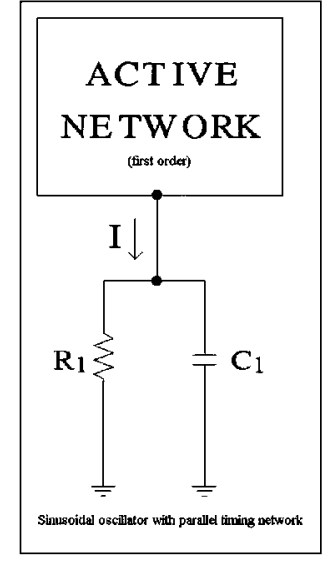

(a)

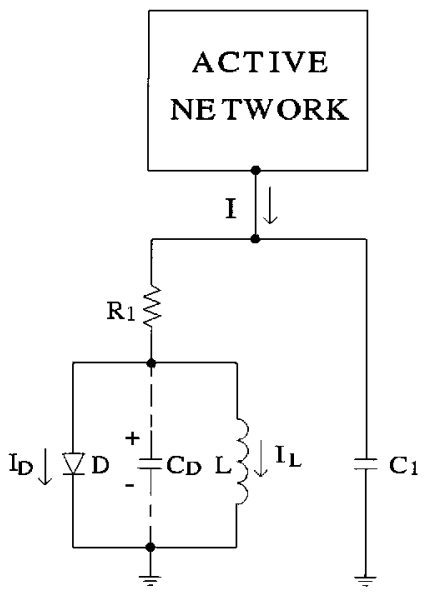

(b)

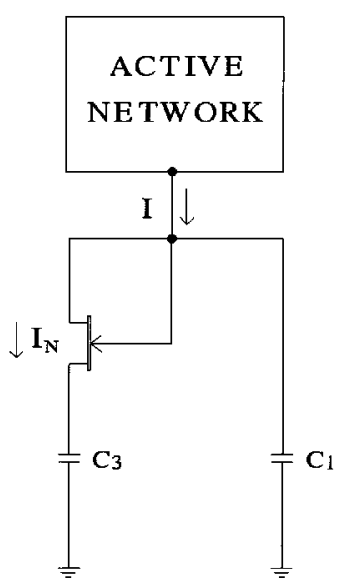

(c)
Fig. 1. Class-I generic circuit-independent oscillators: (a) sinusoidal oscillator with a parallel $R C$ network, (b) chaotic oscillator using a D-L composite, and (c) chaotic oscillator using a FET-C composite.

\section{A. Sinusoidal Oscillator}

A general second-order $R C$ sinusoidal oscillator has the following state space representation:

$$
\left[\begin{array}{c}
\dot{V}_{C 1} \\
\dot{V}_{C 2}
\end{array}\right]=\left[\begin{array}{ll}
a_{11} & a_{12} \\
a_{21} & a_{22}
\end{array}\right]\left[\begin{array}{l}
V_{C 1} \\
V_{C 2}
\end{array}\right]
$$

where $V_{C 1}$ and $V_{C 2}$ are the voltages across its two capacitors. The condition and frequency of oscillation are given respectively by

$$
a_{11}+a_{22}=0 \text { and } \omega_{0}=\sqrt{a_{11} a_{22}-a_{12} a_{21}} .
$$

Now consider Fig. 1(a) where some first-order active network (containing $C_{2}$ ) supplies a current $I$ to the $R_{1} C_{1}$ branch. This current is in general a function of the two state variables $\left(V_{C 1}\right.$ $\left.\& V_{C 2}\right)$ and can be expressed as

$$
I_{ \pm}= \pm g_{1} V_{C 1} \mp g_{2} V_{C 2}
$$

where $g_{1}$ and $g_{2}$ are constant transconductances. Since the oscillator is active, either $g_{1}$ or $g_{2}$ must be negative. Noting that the frequency of oscillation $\omega_{0}$ is generally equal to $\sqrt{n} / R_{1} C_{1}$, where $n$ is a multiplication factor, and by applying the con- 
ditions of (2), the state equations describing Fig. 1(a) can be written in matrix form as

$$
\left[\begin{array}{c}
\dot{V}_{C 1} \\
\dot{V}_{C 2}
\end{array}\right]=\frac{1}{C}\left[\begin{array}{cc} 
\pm g_{1}-g & \mp g_{2} \\
\frac{n g^{2}+\left( \pm g_{1}-g\right)^{2}}{ \pm g_{2}} & g \mp g_{1}
\end{array}\right]\left[\begin{array}{l}
V_{C 1} \\
V_{C 2}
\end{array}\right]
$$

where $g=1 / R_{1}$ and we choose $C_{1}=C_{2}=C$. From (4), it can be seen that the condition for oscillation is satisfied and that $\omega_{0}=g \sqrt{n} / C$.

By introducing the following variables: $\tau=t g_{2} / C, X=$ $V_{C 1} / V_{\text {ref }}, Y=V_{C 2} / V_{\text {ref }}, K_{1}=g_{1} / g_{2}$ and $K_{2}=g / g_{2}$, where $V_{\text {ref }}$ is an arbitrary voltage normalization constant, the dimensionless form of (4) (apart from the term $\epsilon$ ) becomes

$$
\left[\begin{array}{l}
\dot{X} \\
\dot{Y}
\end{array}\right]=\left[\begin{array}{cc} 
\pm K_{1}-K_{2} \pm \epsilon & \mp 1 \\
\pm\left[n K_{2}^{2}+\left( \pm K_{1}-K_{2}\right)^{2}\right] & K_{2} \mp K_{1}
\end{array}\right]\left[\begin{array}{l}
X \\
Y
\end{array}\right]
$$

Note that the frequency of oscillation can be varied using $n$ without affecting the condition of oscillation.

To guarantee that oscillations start, practical oscillators need to have a control parameter to compensate for any losses that may cause the condition for oscillation not to be satisfied. This control parameter is represented in (5) by the small error factor $\epsilon$, which is also responsible for moving the pair of complex conjugate eigenvalues admitted by (5) between the left and the right half-planes. For example, the classical equal-R equal-C Wien-bridge oscillator requires an amplifier with gain $K=3$, in order to start oscillations. By writing $K=3+\Delta K$, one can identify that in this case $\epsilon=\Delta K$.

Note that once oscillation starts, an amplitude control mechanism is needed to stabilize the amplitude of oscillation. This mechanism can be a nonlinear voltage or current-controlled device inserted in the feedback path, or it can simply be the nonlinearity of the active device employed (ex. the saturation-type voltage transfer characteristic of an op amp). Consequently, (5) cannot alone model the behavior of a sinusoidal oscillator since it represents a linear system. However, it does model correctly the function performed by the sinusoidal oscillator engine within the chaotic oscillator structures introduced in the following sections. We emphasize that the generation of chaos is a result of linking sinusoidal oscillators to simple nonlinear composite devices and is associated only with the nonlinear characteristics of these devices and not with any amplitude control mechanism of the sinusoidal oscillator, which is actually not needed in this case. The same nonlinear characteristics responsible for chaos generation guarantee bounded oscillations.

\section{B. Chaotic Oscillator Structure Using a Diode-Inductor Composite}

Consider the configuration shown in Fig. 1(b) which is derived from that of Fig. 1(a) by inserting a diode-inductor (D-L) composite [17] in series with $R_{1}$. The switching action of the diode depends on the voltage across the parasitic transit capacitor $C_{D}$ [27]. Hence, the D-L composite in Fig. 1(b) is described by

$$
\begin{aligned}
L \dot{I}_{L} & =V_{C D} \\
C_{D} \dot{V}_{C D} & =\left(V_{C 1}-V_{C D}\right) / R_{1}-I_{L}-I_{D}
\end{aligned}
$$

and

$$
I_{D}= \begin{cases}g_{D}\left(V_{C D}-V_{\gamma}\right), & V_{C D} \geq V_{\gamma} \\ 0, & V_{C D}<V_{\gamma}\end{cases}
$$

$I_{D}$ is the nonlinear diode current, while $g_{D}$ and $V_{\gamma}$ are the diode forward conduction transconductance and voltage drop respectively.

In addition to the normalization used to derive (5), and by introducing $Z=I_{L} /\left(g_{2} V_{\text {ref }}\right), V=V_{C D} / V_{\text {ref }}, \beta=C / g_{2}^{2} L$, $\varepsilon_{c}=C_{D} / C, K_{D}=g_{D} / g_{2}$, the state space representation of the chaotic oscillator structure of Fig. 1(b) can be derived as

$$
\begin{aligned}
{\left[\begin{array}{c}
\dot{X} \\
\dot{Y} \\
\dot{Z} \\
\varepsilon_{c} \dot{V}
\end{array}\right]=} & {\left[\begin{array}{crrc}
K_{1}-K_{2} \pm \epsilon & -1 & 0 & K_{2} \\
n K_{2}^{2}+\left(K_{1}-K_{2}\right)^{2} & K_{2}-K_{1} & 0 & 0 \\
0 & 0 & 0 & \beta \\
K_{2} & 0 & -1 & -K_{2}-a
\end{array}\right] } \\
& \cdot\left[\begin{array}{c}
X \\
Y \\
Z \\
V
\end{array}\right]+\left[\begin{array}{l}
0 \\
0 \\
0 \\
a
\end{array}\right]
\end{aligned}
$$

and

$$
a= \begin{cases}K_{D}, & V \geq 1 \\ 0, & V<1\end{cases}
$$

Here, we have chosen $V_{\text {ref }}=V_{\gamma}$ and $I=I_{+}$[refer to (3)].

Although the system described by (7) is a fourth-order system, it is effectively living in a three-dimensional subspace as the value of transit capacitor $C_{D}$ is much smaller compared to the other two capacitors in the system $\left(\varepsilon_{c} \rightarrow 0\right)$. We emphasize the fact that the internal structure of the first-order active network remains irrelevant so long as it supplies the current $I$, generally expressed by (3).

By comparing (5) with (7), the evolution of the two-dimensional sinusoidal oscillator dynamics into a higher dimensional state-space can be clearly recognized. Stretching of the trajectories is dominated by the unstable second-order oscillator in the $X-Y$ plane. As oscillations grow in the $X-Y$ plane, energy is continuously transferred to the nonlinear $Z-V$ subsystem through the state variable $X$ with a transfer coefficient $K_{2} / \varepsilon_{c}$. This results in a rapid build-up of the voltage across the diode which eventually switches on $(V>1)$ and dissipates the energy stored in the inductor. The voltage across the diode is sensed by the oscillator with a sensing coefficient equal to $K_{2}$ and the strong dissipation of the energy in the inductor causes the oscillations in the $X-Y$ plane to decay. However, before these oscillations die completely, the diode switches off again $(V<1)$. This repeated stretching and folding of the trajectories provides the necessary mechanism for chaos generation.

Numerical integration of (7) was performed ${ }^{1}$ using the set of parameter values $K_{1}=2, K_{2}=1, K_{D}=50, \varepsilon_{c}=0.01$, $n=0.1, \epsilon=-0.35$ and $\beta=1$. Fig. 2(a) and (b) show the resulting $X-Y$ and $X-Z$ projections of the chaotic attractor respectively. In the upper left corner of Fig. 2(a), the limit cycles observed when (7) is integrated after setting $\epsilon=\varepsilon_{c}=0$ are shown. These limit cycles correspond to the three cases $\left(K_{1}, K_{2}\right)=(2,1),(1,1)$, and $(1,2)$, respectively. It is clear that $K_{1}$ and $K_{2}$ define the phase relation in the $X-Y$ plane.

\footnotetext{
${ }^{1}$ An adaptive-step Runge-Kutta algorithm was used. Note the stiff nature of the differential equations due to the small value of $\varepsilon_{c}$.
} 


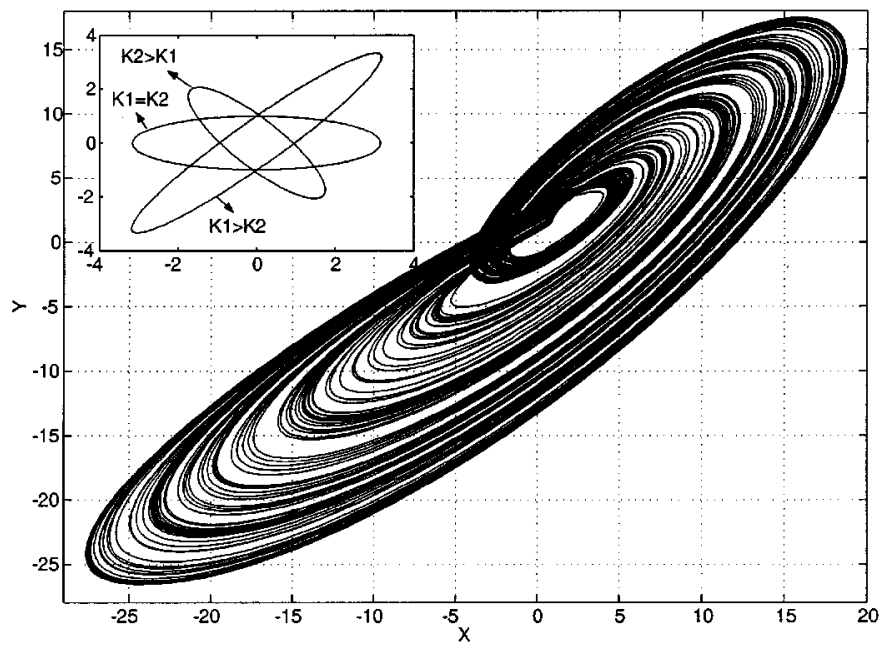

(a)

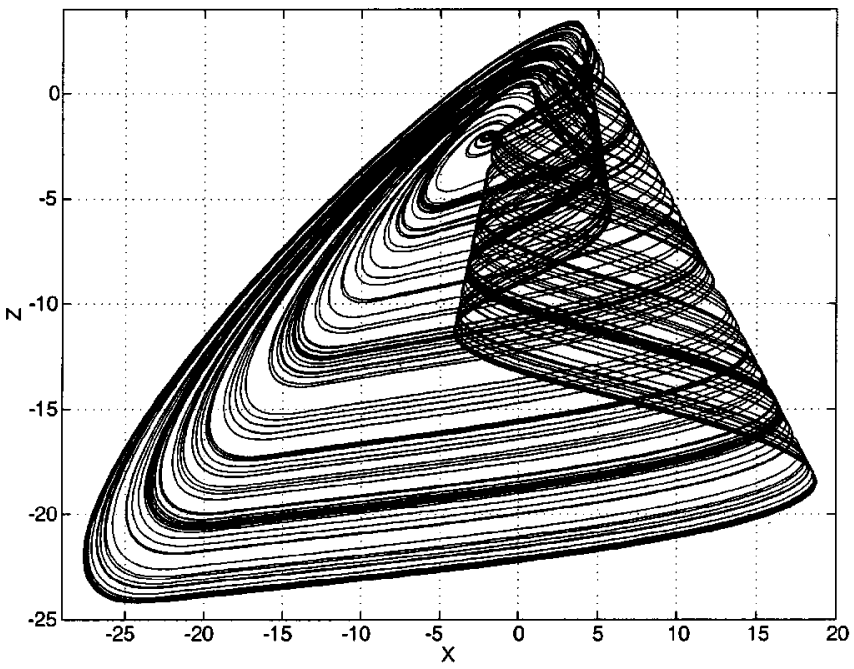

(b)

Fig. 2. Numerical simulation results of (7) $\left(K_{1}=2, K_{2}=1, K_{D}=50, \epsilon=-0.35, \varepsilon_{c}=0.01, n=0.1, \beta=1\right.$ ): (a) $X-Y$ phase projection and (b) $X-Z$ phase trajectory.

In particular, the case $K_{1}>K_{2}$ results in a clockwise phase rotation while $K_{1}<K_{2}$ results in an anticlockwise phase rotation. It is also clear that the best control parameter (bifurcation parameter) for the system is $\epsilon$, which is also the control parameter of its core sinusoidal oscillator engine. In particular, as $|\epsilon|$ is increased further away from its theoretical value required to start oscillations $(\epsilon=0)$ in the direction which causes the pair of complex conjugate eigenvalues to move further into the right half-plane, a period-doubling route to chaos is excited.

It can be shown that the system described by (7) has a single equilibrium point at the origin. This equilibrium point is real in the region $V<1$ and virtual in the region $V \geq 1$ (meaning that it lies outside this region) [28]. The following eigenvalues were calculated at the equilibrium point using the parameters corresponding to Fig. 2: $(-1.005,-100,0.324 \pm j 0.585)$ in the region $V<1$ and $(-0.002,-5100,-0.165 \pm j 0.635)$ in the other region. Thus, the equilibrium point at the origin is an unstable focus.

Several circuit-design examples that follow the structure of Fig. 1(b) can be found in [10], [12] and [29].

\section{Chaotic Oscillator Structure Using a FET-Capacitor Composite}

Consider the structure shown in Fig. 1(c) which is derived from that of Fig. 1(a) by replacing $R_{1}$ with a FET-capacitor $\left(\right.$ FET-C ${ }_{3}$ ) composite [17]. The FET-C is a first-order passive composite with a diode-connected FET. In Fig. 1(c), it is modeled by

$$
C_{3} \dot{V}_{C 3}=I_{N}
$$

and

$$
I_{N}= \begin{cases}g_{N}\left(V_{C 1}-V_{C 3}\right), & V_{C 1}-V_{C 3} \geq V_{P} \\ g_{N} V_{P}, & V_{C 1}-V_{C 3}<V_{P}\end{cases}
$$

where $I_{N}$ is the nonlinear FET current, $g_{N}$ is the FET operating point transconductance, and $V_{P}$ is a negative pinch-off voltage.
By introducing the dimensionless variables $Z=V_{C 3} / V_{\text {ref }}$ and $K_{N}=g_{N} / g_{2}$, in addition to those used to derive (5), the state space representation of Fig. 1(c) is given by

$$
\left[\begin{array}{c}
\dot{X} \\
\dot{Y} \\
\dot{Z}
\end{array}\right]=\left[\begin{array}{ccc}
-K_{1}-a \pm \epsilon & 1 & a \\
-n K_{N}^{2}-K_{1}^{2} & K_{1} & 0 \\
a & 0 & -a
\end{array}\right]\left[\begin{array}{c}
X \\
Y \\
Z
\end{array}\right]+\left[\begin{array}{c}
-b \\
0 \\
b
\end{array}\right]
$$
and

$$
(a, b)= \begin{cases}\left(K_{N}, 0\right) & X-Z \leq 1 \\ \left(0, K_{N}\right) & X-Z>1 .\end{cases}
$$

Here, we have chosen $V_{\mathrm{ref}}=V_{P}, C_{1}=C_{2}=C_{3}=C$ [recall (4)] and $I=I_{-}$[recall (3)].

Numerical integration of (9) was performed using a fourth-order Runge-Kutta algorithm with a 0.001 time step taking $K_{1}=1, K_{N}=2, n=0.2$, and $\epsilon=-0.3 . X-Y$ and $Y-Z$ projections of the observed chaotic attractor are shown in Fig. 3(a) and (b) respectively. In the upper left corner of Fig. 3(a) the limit cycle obtained when $\epsilon=a=0$ is plotted. It can be shown that this system has an equilibrium point $\left(x_{0}, y_{0}, z_{0}\right)=(0,0, b / a)$. Hence, the origin is a real equilibrium point in the region $X-Z \leq 1$ with the set of eigenvalues $(-4.546,0.123 \pm j 0.453)$. In the region $X-Z>1$, the eigenvalues are $(0,-0.65 \pm j 1.174)$. The origin is an unstable saddle focus.

Note from (9) that the condition $a=0$ (FET off) implies that the three-dimensional system collapses onto the two-dimensional $X-Y$ plane. In this case, the oscillator remains linked to the nonlinear subsystem (the FET- $C_{3}$ composite) through a constant current source $\left(I_{N}=g_{N} \cdot V_{P}\right)$ which continuously charges $C_{1}$ by discharging $C_{3}$. The negative pinch-off voltage $V_{P}$ guarantees this discharge process. Eventually, the gate-to-source voltage of the FET $\left(V_{C 1}-V_{C 3}\right)$ exceeds $V_{P}$ and the FET is turned on again. One can show that the characteristic equation of (9) always admits a negative real eigenvalue for all values of $a>0$. In addition, when the condition $n<2 K_{1} / K_{N}$ is satisfied, a pair of complex conjugate eigenvalues in the right half-plane is always admitted. 


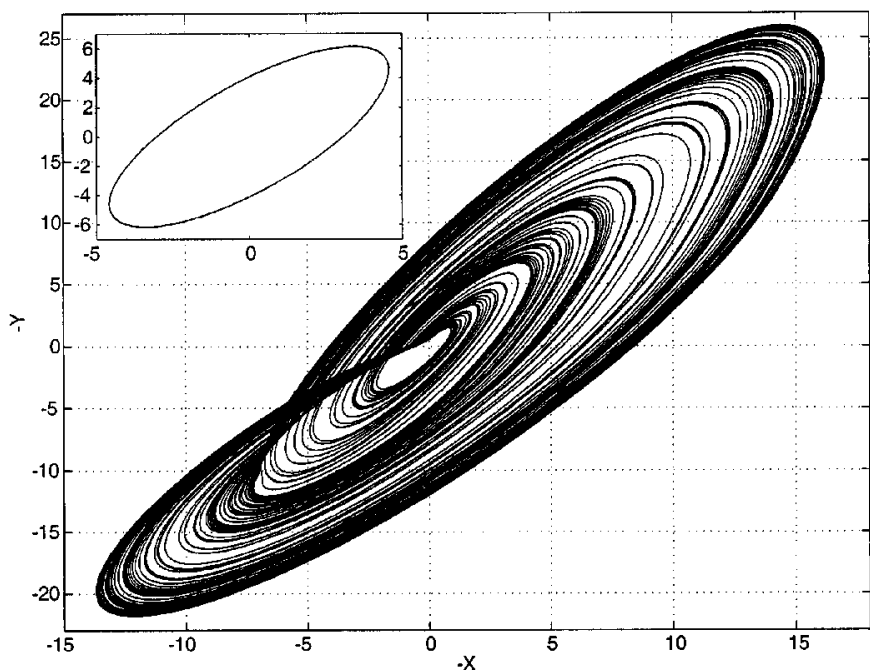

(a)

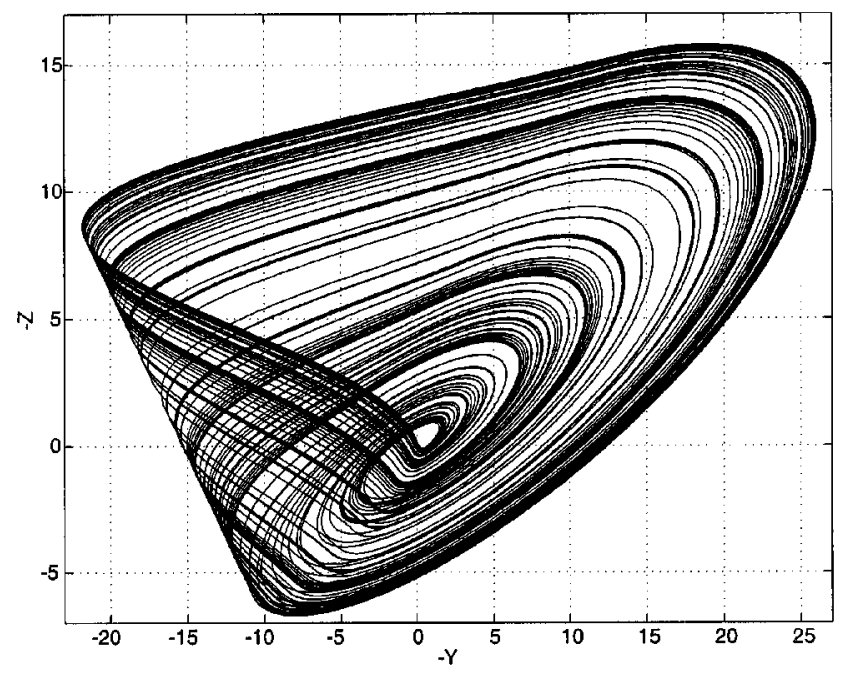

(b)

Fig. 3. Results of numerical integration of (9) $\left(K_{1}=1, K_{N}=2, n=0.2\right.$, $\epsilon=-0.3$ ): (a) $X-Y$ projection and (b) $Y-Z$ projection.

\section{Class II ChaOtic Oscillators}

The chaotic oscillators of this class are based on the general sinusoidal oscillator shown in Fig. 4(a) characterized by having a separate series $R_{1} C_{1}$ network, which is also a common feature in many oscillators [23]-[26]. This series network can either be driven by a current $I$, generally given by (3), or by a voltage $V_{S}$ generally given as $V_{S}= \pm K_{1} V_{C 1} \mp K_{2} V_{C 2}$. Thus, the function of the first-order active network (containing $C_{2}$ ) is either to supply $I$ or maintain $V_{S}$. In the following sections we show how a chaotic oscillator can be derived in both cases.

\section{A. Sinusoidal Oscillator}

Consider the case where the $R_{1} C_{1}$ network is current-driven. By recalling (1)-(3), Fig. 4(a) can then be described by

$$
\left[\begin{array}{c}
\dot{V}_{C 1} \\
\dot{V}_{C 2}
\end{array}\right]=\frac{1}{C}\left[\begin{array}{cc} 
\pm g_{1} & \mp g_{2} \\
\frac{n g^{2}+g_{1}^{2}}{ \pm g_{2}} & \mp g_{1}
\end{array}\right]\left[\begin{array}{l}
V_{C 1} \\
V_{C 2}
\end{array}\right]
$$

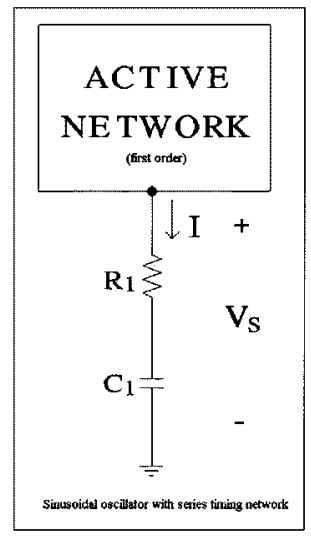

(a)

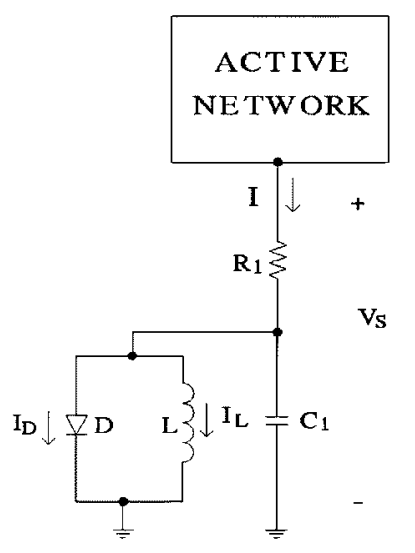

(b)

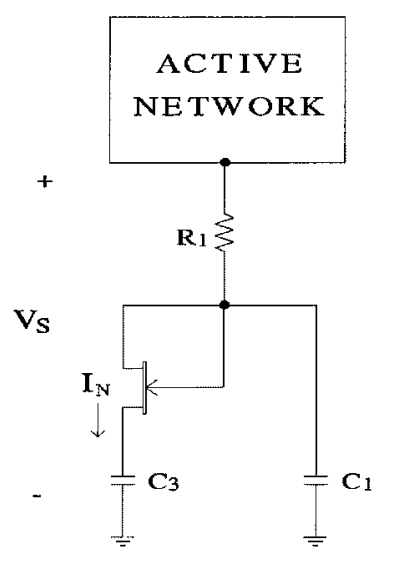

(c)
Fig. 4. Class-II generic circuit-independent oscillators: (a) sinusoidal oscillator with a series $R C$ network, (b) chaotic oscillator using a D-L composite, and (c) chaotic oscillator using a FET-C composite.

where $C_{1}=C_{2}=C$ and $g=1 / R_{1}$. It can be seen that the condition for oscillation is satisfied and the frequency of oscillation is $\omega_{0}=g \sqrt{n} / C$.

Adopting the same dimensionless variables used to derive (5), (10) becomes

$$
\left[\begin{array}{c}
\dot{X} \\
\dot{Y}
\end{array}\right]=\left[\begin{array}{cc} 
\pm K_{1} \pm \epsilon & \mp 1 \\
\pm\left[n K_{2}^{2}+K_{1}^{2}\right] & \mp K_{1}
\end{array}\right]\left[\begin{array}{c}
X \\
Y
\end{array}\right]
$$

where $\epsilon$ is the error control parameter used to guarantee that oscillations start. Note that $K_{2}$ appears here as a frequency multiplication factor and does not affect the condition for oscillation.

In the case where the $R_{1} C_{1}$ network is voltage-driven by $V_{S}$, Fig. 4(a) is described by

$$
\left[\begin{array}{c}
\dot{X} \\
\dot{Y}
\end{array}\right]=\left[\begin{array}{cc} 
\pm K_{1}-1 \pm \epsilon & \mp K_{2} \\
\frac{n+\left( \pm K_{1}-1\right)^{2}}{ \pm K_{2}} & 1 \mp K_{1}
\end{array}\right]\left[\begin{array}{l}
X \\
Y
\end{array}\right] .
$$

Here, the time normalization constant $\tau$ is taken as $t g / C$ instead of $t g_{2} / C$.

\section{B. Chaotic Oscillator Using a D-L Composite}

The structure shown in Fig. 4(b) is obtained from that of Fig. 4(a) by adding a D-L composite in parallel with $C_{1}$. The 


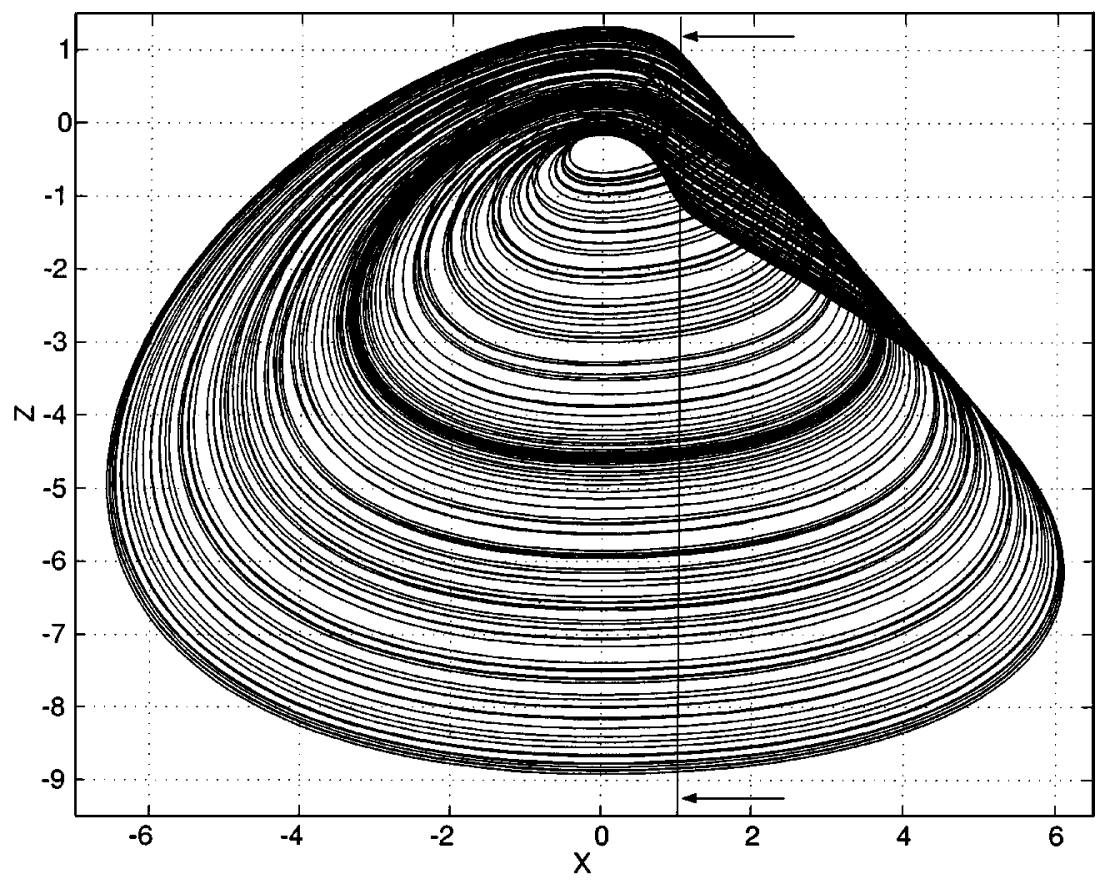

Fig. 5. $X-Z$ trajectory resulting from numerically integrating (13) $\left(K_{1}=2, K_{2}=0.1, K_{D}=3, n=1, \beta=1, \epsilon=0.32\right)$.

series network is current-driven and the configuration is thus described by

$$
\begin{aligned}
{\left[\begin{array}{c}
\dot{X} \\
\dot{Y} \\
\text { and }
\end{array}\right] } & =\left[\begin{array}{ccc}
-K_{1}-a \pm \epsilon & -1 & -1 \\
n K_{2}^{2}+K_{1}^{2} & -K_{1} & 0 \\
\beta & 0 & 0
\end{array}\right]\left[\begin{array}{l}
X \\
Y \\
Z
\end{array}\right]+\left[\begin{array}{l}
a \\
0 \\
0
\end{array}\right] \\
a & = \begin{cases}K_{D}, & X \geq 1 \\
0, & X<1 .\end{cases}
\end{aligned}
$$

The same settings that were used to derive (7) were also used in deriving (13) with the choice of $V_{\text {ref }}=V_{\gamma}$ and $I=I_{+}$. Note that the voltage across the inductor appears across $C_{1}$ which dominates the diode transit capacitance $C_{D}$.

By comparing (11) and (13), the role of the sinusoidal oscillator in the $X-Y$ plane can be identified. Assuming that the diode is initially off ( $a=0)$, oscillations will continue to grow in the $X-Y$ plane transferring energy to the nonlinear subsystem via the state variable $X$ with a transfer coefficient $\beta$. This results in a continuous increase of $Z$. However, the oscillator in the $X-Y$ plane is linked to the nonlinear subsystem through the term $-Z$. Hence, the increasing $Z$ tends to slow down the growth of oscillation in the $X-Y$ plane by reducing the amount of current charging $C_{1}$. Eventually, when $V_{C 1}$ reaches $V_{\gamma}$, the diode switches on dissipating strongly the energy transferred from the oscillator and rapidly discharging $C_{1}$. The growth of oscillations is then reversed into an oscillation decay until the diode switches off again.

The $X-Z$ projection of the chaotic attractor obtained via numerical integration of (13) is shown in Fig. 5. The switching plane at $X=1$ is marked. The parameter values in this case are $K_{1}=2, K_{2}=0.1, K_{D}=3, n=1, \beta=1$, and $\epsilon=0.32$. The period-doubling cascade starts at $\epsilon \approx 0.235$. The system described by (13) has a single equilibrium point at the origin with the set of eigenvalues $(-0.362,-1.159 \pm j 2.047)$ in the region $X \geq 1$ and the set $(-1.073,0.696 \pm j 1.174)$ in the region $X<1$. One can show that (13) always admits a negative real eigenvalue. In addition, a complex conjugate pair is always admitted in the right-half plane when $a=0$. The condition $a>1$ guarantees a complex conjugate pair in the left half-plane.

\section{Generation of Chaos Using a FET-C Composite}

A FET-C composite can be placed in parallel with capacitor $C_{1}$ in the oscillator of Fig. 4(a). The resulting structure is shown in Fig. 4(c). Since the case of a current-driven port is similar to that described in Section II-C, we consider here the case of a voltage-driven port with $V_{S}=K_{1} V_{C 1}-K_{2} V_{C 2}$. In this case the structure of Fig. 4(c) can be described by

$$
\left[\begin{array}{c}
\dot{X} \\
\dot{Y} \\
\dot{Z}
\end{array}\right]=\left[\begin{array}{ccc}
K_{1}-1-a \pm \epsilon & -K_{2} & a \\
\frac{n+\left(K_{1}-1\right)^{2}}{K_{2}} & 1-K_{1} & 0 \\
a & 0 & -a
\end{array}\right]\left[\begin{array}{c}
X \\
Y \\
Z
\end{array}\right]+\left[\begin{array}{c}
-b \\
0 \\
b
\end{array}\right]
$$

where $a$ and $b$ are as given by (9b). The same dimensionless variables used to derive (9) are used in (14) with the time normalization constant $\tau$ taken as $t g / C$ instead of $t g_{2} / C$.

By comparing (12) and (14), the evolution of the two-dimensional oscillator dynamics into the three-dimensional space becomes clear. It can also be shown that the characteristic equation of (14) always admits a negative real eigenvalue for all values of $a>0$. In addition, when the condition $K_{1}<1$ is satisfied, a pair of complex conjugate eigenvalues in the right half-plane are always admitted.

When numerically integrating (14) with $K_{1}=0, K_{2}= \pm 1$, $K_{N}=2, n=0.7$, and $\epsilon=-0.3$, the observed trajectories were similar to those shown in Fig. 3(a) and (b) respectively. However, we note that the change of $K_{2}$ from 1 to -1 and vice versa produces a mirror image of the attractor without changing 


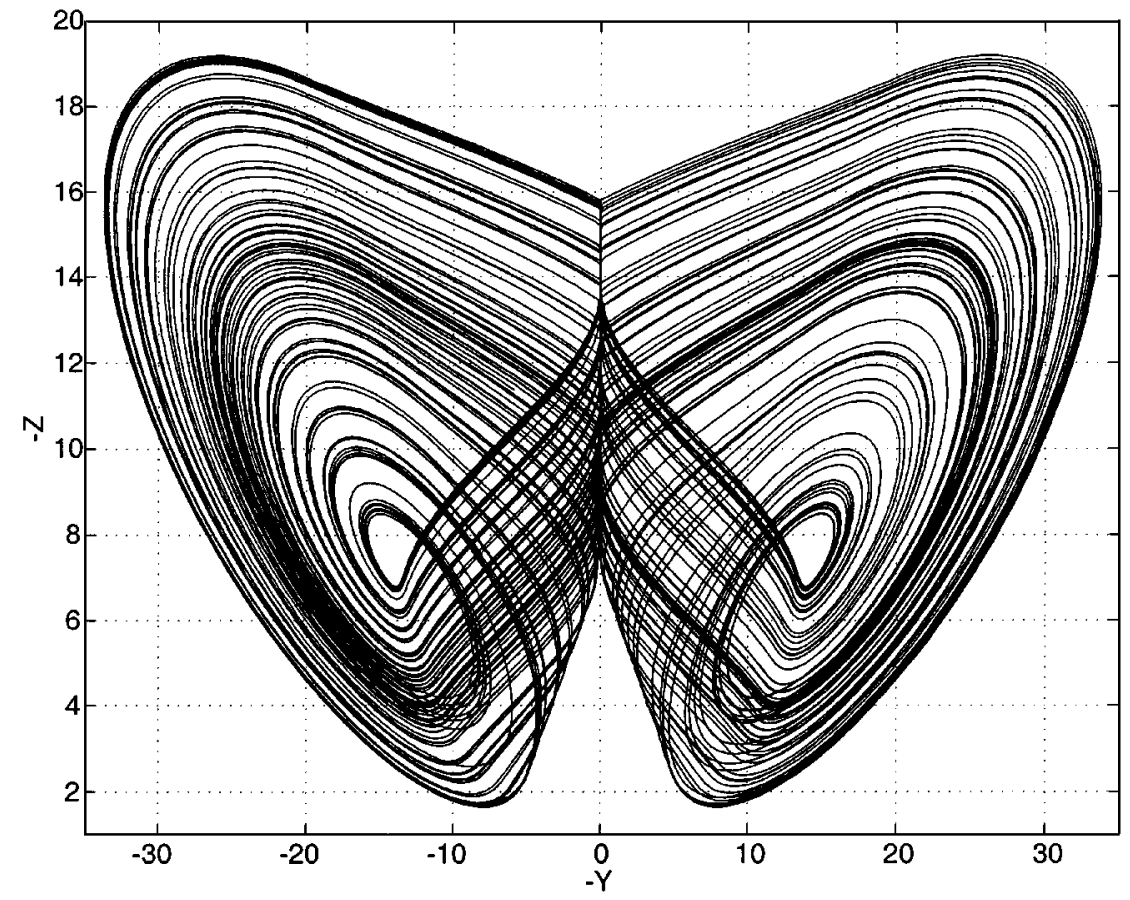

Fig. 6. $Y-Z$ trajectory observed after modifying (14) such that $K_{2}=1$ if $Y \geq 0$ and $K_{2}=-1$ if $Y<0\left(K_{1}=0, K_{N}=2, n=0.9, \epsilon=-0.2, m=5\right)$.

its dynamics. In fact, the characteristic equation of (14) is independent of $K_{2}$. Hence, the eigenvalue pattern is not affected by its value.

To demonstrate this interesting observation, we modify (14) such that $K_{2}$ continuously switches between 1 and $-1 . K_{2}$ is set to 1 if $Y \geq 0$ and is set to -1 if $Y<0\left(K_{2} Y=|Y|\right.$ is an even-symmetrical nonlinearity). In addition, a constant $m$ is used to displace the trajectories along the $Y$ axis such that they either lie in the negative half space or the positive half-space when $K_{2}$ is switched. Accordingly, $\dot{X}$ becomes $\dot{X}+m$. The $Y-Z$ trajectory observed in this case is shown Fig. 6. Here, $K_{1}=0, K_{N}=2, n=0.9, \epsilon=-0.2$ and $m=5$.

The similarity between this chaotic attractor and the wellknown Lorenz butterfly attractor is apparent. In fact, we argue that one of the state variables of the Lorenz system performs a function similar to that of the switching constant $K_{2}$. This is explained in the following section.

\section{Modified Lorenz System}

Consider the well-known Lorenz system of differential equations given by [22]

$$
\begin{aligned}
\dot{X} & =a(Y-X) \\
\dot{Y} & =(b-Z) X-Y \\
\dot{Z} & =X Y-c Z
\end{aligned}
$$

where $a, b$, and $c$ are constants. The two multiplier-type nonlinearities $(X Y$ and $X Z)$ are responsible for the generation of chaos in this system. Circuit realizations of the Lorenz system have always been difficult because of these multipliers [30].

From (15c), it can be seen that when the nonlinear term $X Y$ is sufficiently small, $Z$ scales as $e^{-c t}$, thus decaying with time and allowing the oscillations in the $X-Y$ plane to grow. Eventually, the nonlinear term $X Y$ is no longer small and dominates the term $-c Z$, resulting in a continuous increase of $Z$. This increase reflects back to the $X-Y$ plane via the nonlinear term $(b-Z) X$ in (15b). Actually, $b$ can be thought of as a threshold level since the term $(b-Z)$ changes its sign at the threshold $Z=b$. As $Z$ exceeds $b$, oscillation growth is stopped and reversed into a decay. We note that the term $(b-Z)$ alone can guarantee this reverse process when it changes sign. We also note that the $X-Z$ projection of the Lorenz attractor is symmetrical with respect to the $X$ axis and that $Z$ is always positive. This implies that for a small enough threshold $b$, the term $(b-Z)$ will always change sign. Hence, the role played by the state variable $X$ in the nonlinear term $(b-Z) X$ is similar to that played by $K_{2}$ in the system described by (14). We thus suggest modifying (15b) to read

$$
\dot{Y}=K(b-Z)
$$

and

$$
K=\operatorname{sgn}(X)=\left\{\begin{array}{rl}
1 & X \geq 0 \\
-1 & X<0
\end{array}\right.
$$

Note that we have also eliminated the term $-Y$ from (15b) since it is not necessary.

By observing that the $Z$ signal is always positive, we conclude that the nonlinear term $X Y$ in (15c) is not particular. It can be replaced by other terms which will ensure that $Z$ remains positive. Such terms might be $X^{2},|X|$ or simply $K X$, where $K$ is the switching constant given in (16). In conclusion, we propose the following system, which has similar qualitative dynamics to the Lorenz system but is multiplier-free:

$$
\left[\begin{array}{c}
\dot{X} \\
\dot{Y} \\
\dot{Z}
\end{array}\right]=\left[\begin{array}{ccc}
-a & a & 0 \\
0 & 0 & -K \\
K & 0 & -c
\end{array}\right]\left[\begin{array}{c}
X \\
Y \\
Z
\end{array}\right]+\left[\begin{array}{c}
0 \\
b K \\
0
\end{array}\right]
$$

where $K$ is as given in (16). 


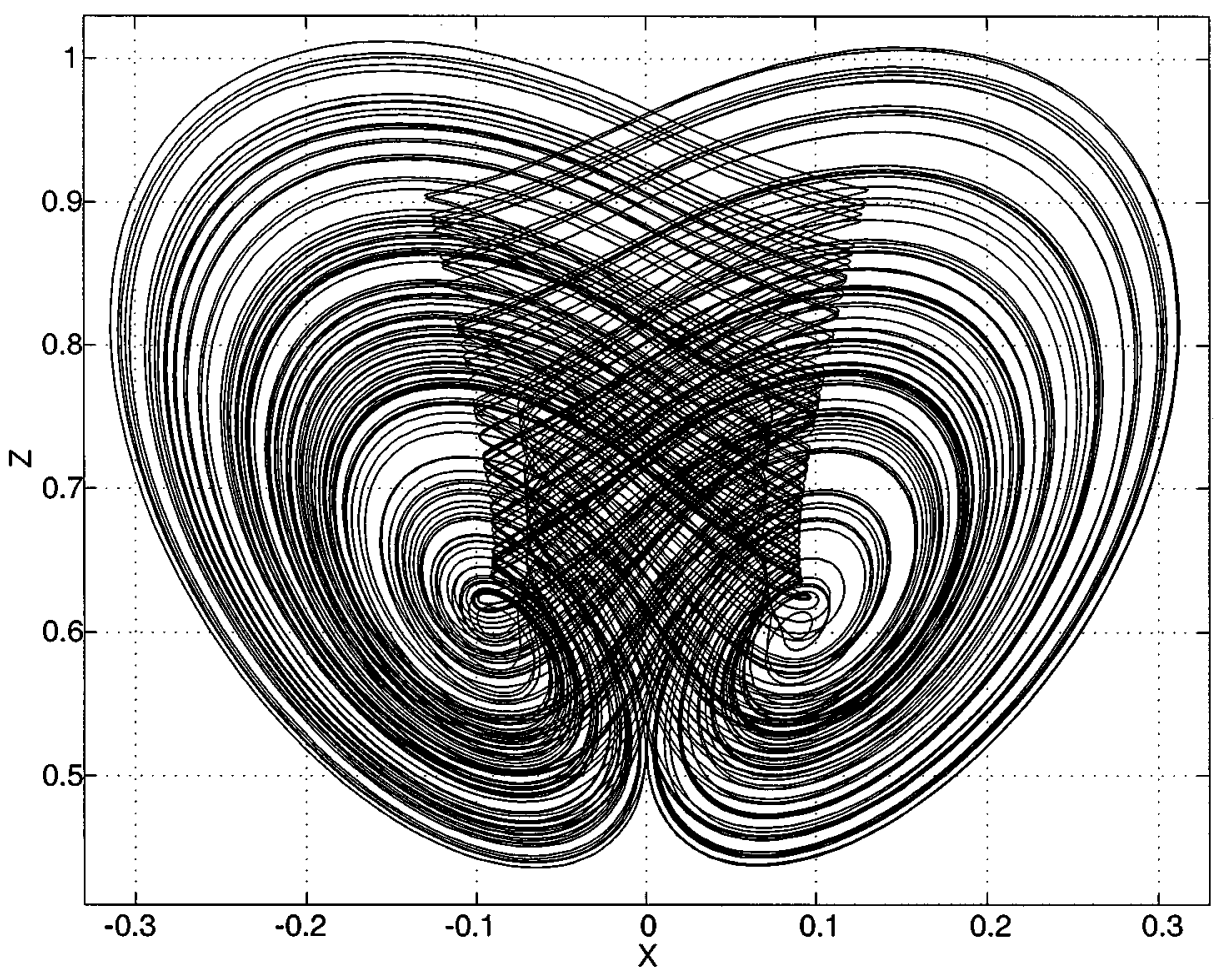

(a)

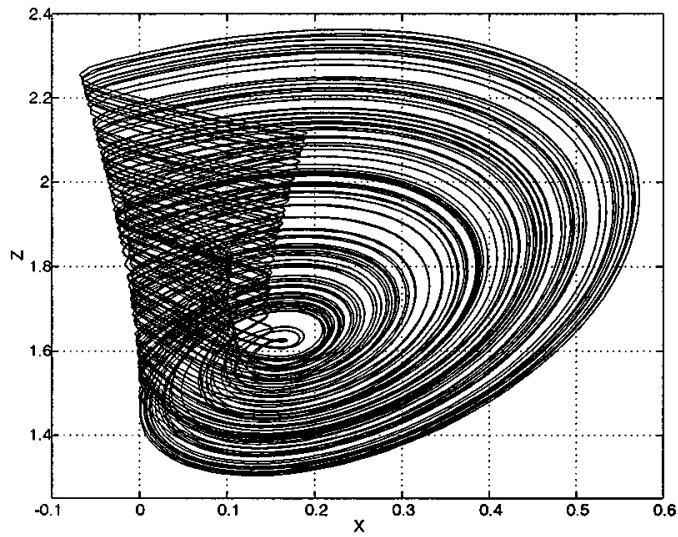

(b)

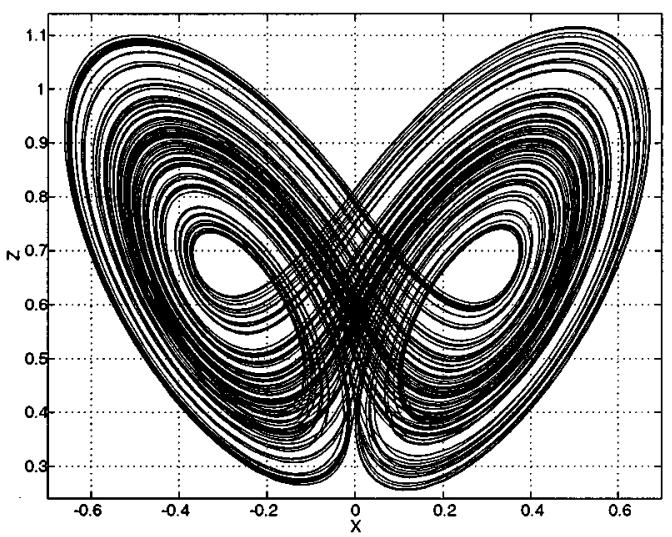

(c)

Fig. 7. Projections of the butterfly attractor in the $X-Z$ plane observed from our proposed multiplier-free Lorenz system: (a) $a=b=0.6, c=0.15, m=0$, (b) $a=b=0.6, c=0.15, m=1$, and (c) $a=b=0.6, c=0.45, m=0$.

The $X-Z$ projection of the butterfly attractor observed when numerically integrating (17) with $a=b=0.6$ and $c=0.15$ is shown in Fig. 7(a). We can further modify (17) by adding a constant term $m$ to $\dot{Y}$ to become $\dot{Y}+m$. The attractor in Fig. 7(a) corresponds to the case $m=0$ whereas that of Fig. 7(b) corresponds to the case $m=1$. When $m$ equals -1 , the mirror image of the attractor of Fig. 7(b) is obtained. Thus $m$ allows us to observe both wings of the butterfly $(m=0)$ or simply one of the wings alone $(m= \pm 1)$. It also clearly shows that the Lorenz attractor is constructed by merging together two similar chaotic attractors, one of which is the mirror image of the other. The transition from one attractor to the other can be made smoother by increasing the value of the damping constant $c$, as demonstrated in Fig. 7(c) for $c=0.45$.

The way these two attractors are merged together is dictated by the characteristics of the nonlinearity, which in this case is even-symmetrical $(f(X)=K X=|X|)$. Were the nonlinearity to be odd-symmetrical, these two attractors would have been merged after an additional flip operation had been performed to one of them [31], [32]. In general, we conjecture the following.

Conjecture 2: If a chaotic attractor is observed from a system with a symmetrical (odd or even) nonlinearity, then another chaotic attractor can be observed from the anti-symmetrical half of this nonlinearity. The attractor of the symmetrical 
nonlinearity is related directly to two of the attractors of its anti-symmetrical half by one merge operation, one mirror operation, and an additional flip operation if the nonlinearity is odd [33].

The merge operation implies the continuity of the trajectories when the system switches from one half of the nonlinear characteristics to the other. Therefore, studying the nonlinear dynamics of the anti-symmetrical "half attractor" should be sufficient to understand the dynamics of the symmetrical "full attractor." An interesting example that has been reported recently is the quad-screw chaotic attractor consisting of four single-screws or equivalently two double-screws [34], [35], implying the existence of both and odd and an even-symmetrical nonlinearity.

\section{ON The Simplest Possible Chaotic Dynamics}

It is well known that the simplest possible antisymmetric nonlinearity is that of the passive diode (or diode-connected transistor). On the other hand, the simplest possible systems that can admit oscillations are the second-order $L C-r$ current or voltage-controlled negative resistance oscillator, its equivalent $F D N R-R$ oscillator, and the generic $R C$ oscillators shown in Figs. 1(a) and 4(a). Hence, a third-order continuous-time chaotic oscillator where any of these oscillators can be identified as being responsible for stretching the trajectories and where the switching action of a passive device is responsible for folding, will most likely exhibit the simplest possible chaotic dynamics. We conjecture the following.

Conjecture 3: The simplest possible dynamics of an autonomous continuous-time chaotic oscillator are most likely those which can be observed when:

1) the oscillator is described by a third-order system of differential equations;

2) the ON-OFF switching action of a single passive device is the only nonlinearity;

3) the describing equations of second-order subsystem, which admits a pair of unstable complex conjugate eigenvalues in at least one of the regions of operation of the switching device, can be identified.

Examples of chaotic oscillators that satisfy these requirements were given in the previous sections of this article and for example in [36]. We believe that the following third-order canonical model is the simplest possible that captures the essential dynamics of these examples:

$$
-\dddot{X}=\ddot{X}+B \dot{X}+X
$$

and

$$
B= \begin{cases}\alpha_{1}, & f(X, \dot{X}) \geq 1 \\ \alpha_{2}, & f(X, \dot{X})<1 .\end{cases}
$$

The projection of the chaotic attractor observed from (18) in the $\dot{X}-X$ plane is shown in Fig. 8 when $\alpha_{1}=5, \alpha_{2}=0$, and $f(X, \dot{X})=\dot{X}$. The switching plane at $\dot{X}=1$ is marked. The eigenvalues at the equilibrium point are $(-0.207,-0.397 \pm$ $j 2.163)$ when $B=\alpha_{1}$ and $(-1.466,0.233 \pm j 0.796)$ when $B=\alpha_{2}$.

We note the following about the system of (18).

1) The system has a single equilibrium point at the origin.

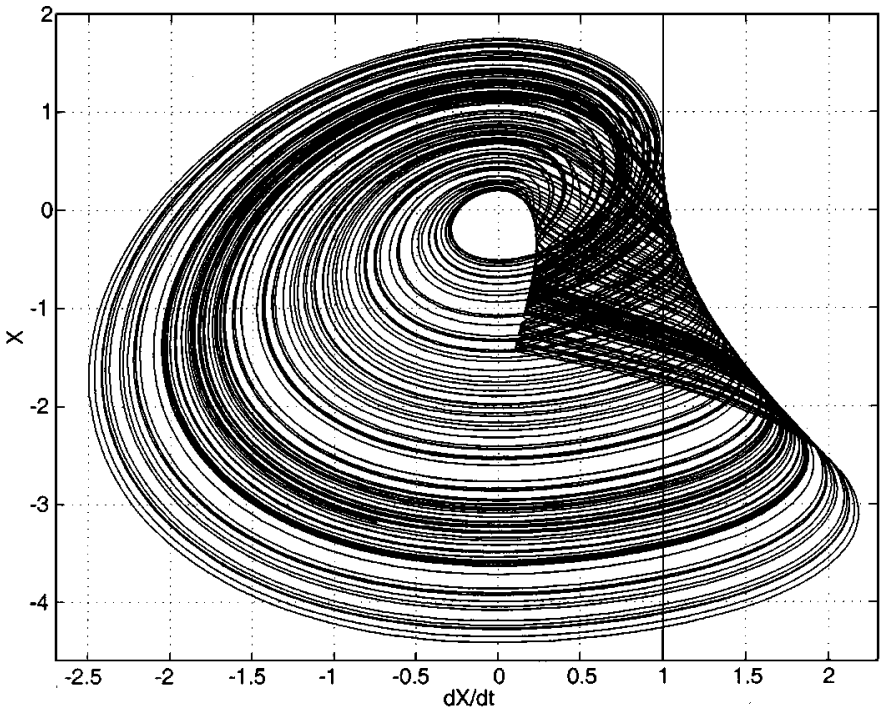

Fig. 8. $\dot{X}-X$ projection of the chaotic attractor of (18) $\left(\alpha_{1}=5, \alpha_{2}=0\right.$, and $f(X, \dot{X})=\dot{X})$.

2) The critical value $B=1$ implies that the system has a pair of complex conjugate eigenvalues located on the imaginary axis. This pair moves into the right half-plane when $B<1$ and into the left-half when $B>1$. Therefore, the conditions $\alpha_{1}>1$ and $\alpha_{2}<1$ guarantee that this pair traverses from one half into the other when $B$ switches between $\alpha_{1}$ and $\alpha_{2}$. These two conditions are necessary for chaos generation.

3) A real eigenvalue located in the left half-plane is always admitted.

4) From our numerical simulations we observe the following.

a) When $\alpha_{2}=1$, the only behavior admitted by the system is a simple limit cycle; this happens independently of the value of $\alpha_{1}$.

b) When $\alpha_{2}>1$, oscillations decay and the system settles at the origin independently of the value of $\alpha_{1}$.

c) When $\alpha_{2}<1$, the system will admit chaos only if $\alpha_{1}>1$. This is a necessary but not sufficient condition. In particular, unbounded oscillations (diverging trajectories) always result if the condition $\epsilon=\Delta_{L H P} / \Delta_{R H P}-P_{L H P} / P_{R H P}<0$ is satisfied where, $\Delta_{L H P}=\alpha_{1}-1, \Delta_{R H P}=1-\alpha_{2}$, $P_{L H P}$ and $P_{R H P}$ are the absolute values of the real parts of the complex conjugate eigenvalues calculated respectively at $B=\alpha_{1}$ and $B=\alpha_{2}$. A limit cycle is born when $\epsilon=0$ and chaos requires that $\epsilon>0$. The larger this quantity, the further the system is pushed toward the chaotic zone. The period-doubling cascade starts at approximately $\epsilon=$ 1.3 .

5) When $B$ is used to map the ON-OFF switching action of practical devices, such as diodes and transistors, this will imply that $\alpha_{2} \rightarrow 0$ (very high resistance OFF region) and $\alpha_{1} \gg 1$ (very low resistance ON region). This means that the behavior most likely to be observed from this system when using these passive devices is chaos. 


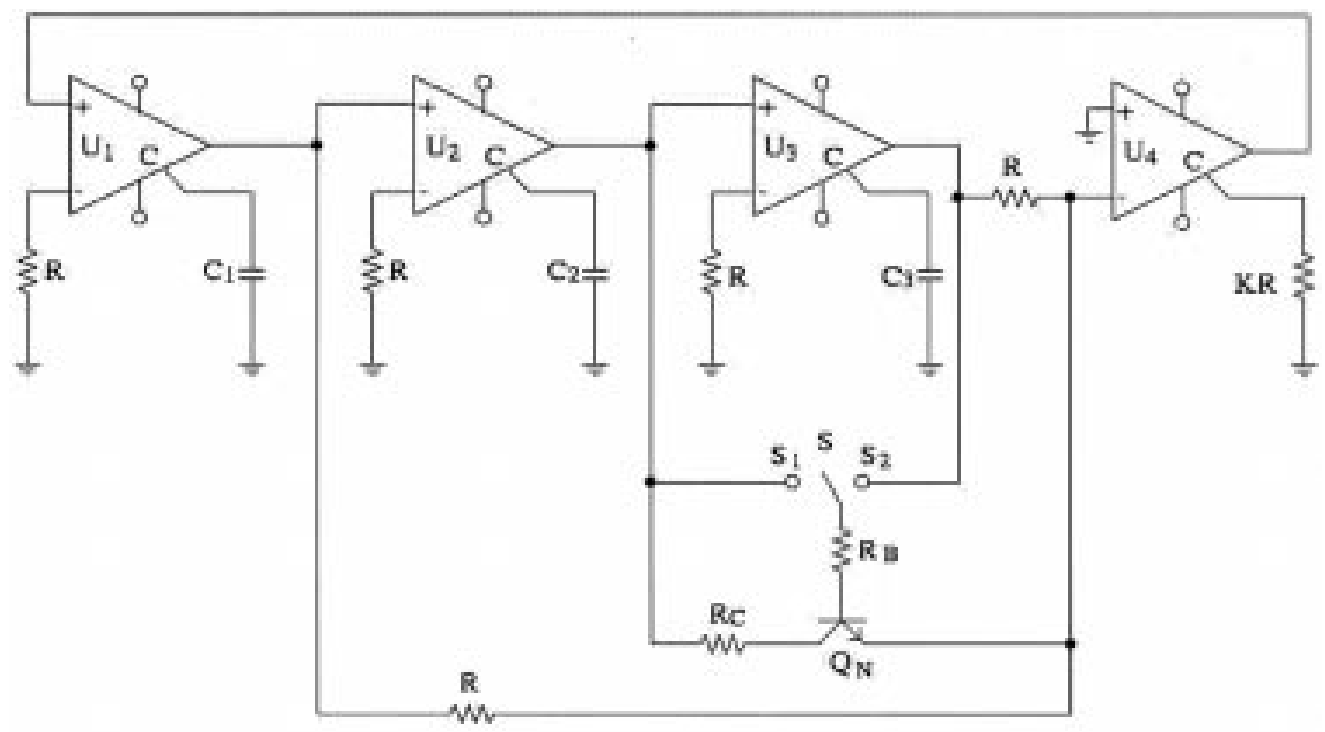

(a)

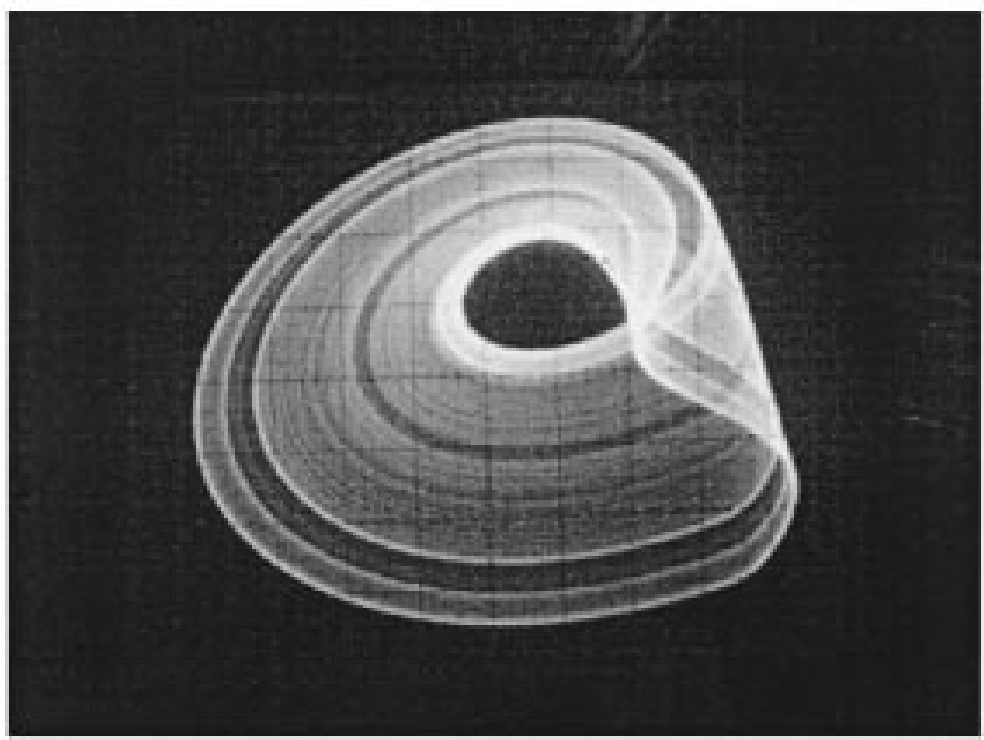

(b)

Fig. 9. Practical realization of (18). (a) Circuit based on an integrator-summer architecture using AD844 current feedback op amps. (b) Experimental $V_{C 2}-V_{C 3}$ trajectory when $S$ is connected to $S_{1}(X$ axis: $0.2 \mathrm{~V} / \mathrm{div}, Y$ axis: $0.2 \mathrm{~V} / \mathrm{div})$.

A straightforward circuit implementation of (18), which comprises three cascaded lossless noninverting integrators followed by an inverting current summer with gain, is shown in Fig. 9(a). The switching action is performed by the bipolar transistor $Q_{N}$. By connecting together the two nodes marked $S$ and $S_{1}$, the switching condition $f(X, \dot{X})$ is equal to $\dot{X}$ and by connecting $S$ to $S_{2}$, it is equal to $X$. Note that the faithful realization of (18) requires the gain $K$ of the inverting summer to equal unity. In this case, the circuit can be tuned using resistor $R_{C}$ (the collector resistance of $Q_{N}$ ) which should equal $R / \alpha_{1}$. However, in practice, it is more convenient to fix the value of $R_{C}$ and to use the gain $K$ as the tuning parameter.
An experimental setup of the circuit was constructed ${ }^{2}$ with $R=1 \mathrm{k} \Omega, C_{1}=C_{2}=C_{3}=1 \mathrm{nF}, R_{B}=1 \mathrm{k} \Omega, R_{C}=100 \Omega$, and a $5 \mathrm{k} \Omega$ pot. was used to tune the gain resistor $K R$. The $V_{C 2}-V_{C 3}(\dot{X}-X)$ phase portrait observed when $f(X, \dot{X})=$ $\dot{X}$ is shown in Fig. 9(b).

\section{ON THE StRUCtURE OF CHAOTIC OsCillators}

In the previous sections, two classes of generic chaotic oscillator structures were designed. Both classes evolve from the

\footnotetext{
${ }^{2} \mathrm{AD} 844 \mathrm{CFOA}$ chips biased with $\pm 9 \mathrm{~V}$ and a Q2N2222 NPN transistor were used.
} 


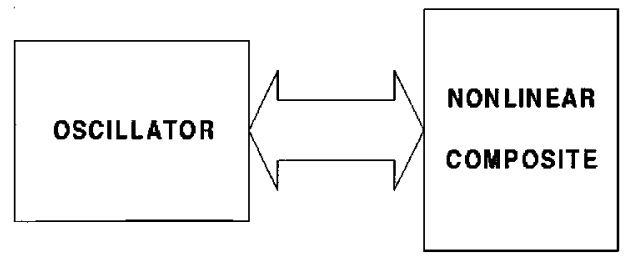

(a)

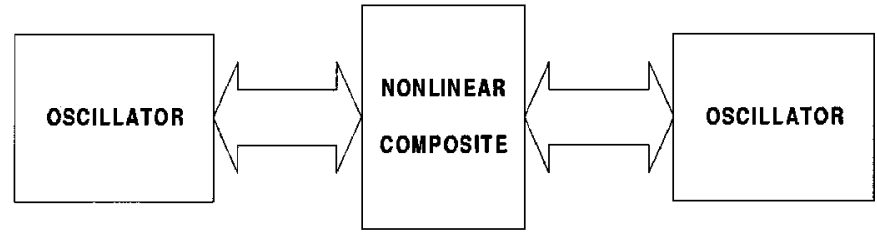

(b)

NON LINEAR COMPOSITE

(c)

Fig. 10. General structures for autonomous chaotic oscillators: (a) low-order oscillators follow the oscillator-nonlinear composite architecture, (b) a class of higher order oscillators can be constructed by coupling sinusoidal (relaxation) oscillators via a nonlinear composite, or by (c) inserting a filter section between the oscillator and the nonlinear composite.

simple dynamics of a sinusoidal oscillator which is coupled to a passive nonlinear composite. Although we have considered the D-L and the FET-C nonlinear composites, other composites can also be introduced. For example, we have used a passive hysteresis nonlinear resistor from those of [37] to generate a class of hysteresis chaotic oscillators [38]. It was shown in [38] that a hysteresis chaotic oscillator is the limit case of another chaotic oscillator when one of its energy storage elements (inductor or capacitor) becomes significantly small, or even a parasitic, such that slow-fast dynamics arise. ${ }^{3}$

In this section we discuss three general architectures into which most autonomous chaotic oscillators can be decomposed. Two of these architectures are particularly suitable for generating higher-order and hyperchaotic oscillators.

\section{A. The Oscillator-Nonlinear Composite}

The structure represented in Fig. 10(a) can be recognized in a large number of autonomous chaotic oscillators. It is composed of two blocks: an oscillator and a nonlinear composite. Interaction between these two blocks is commonly supported by a zero-order link, meaning that it is either a direct link or a resistive one. In order two admit chaos, two conditions are necessary.

- At least one of the two blocks must be active.

- At least three energy storage elements (including parasitic ones) must exist.

In all systems which we have investigated, we found that the oscillator was sinusoidal. A summary of our investigation results is given in Table I. In particular, two examples can be given for a system with a zeroth-order nonlinear composite. These two examples are the chaotic Colpitts oscillator [3] and the chaotic Twin-T oscillator [9], both of which are derived from third-order sinusoidal oscillators. Being of zeroth-order, the nonlinear composite (actually a single nonlinear device) is embedded within the oscillator rather than being linked to it. However, one can in general identify a particular energy-storage element which

\footnotetext{
${ }^{3}$ This is true also for relaxation oscillators which relate to sinusoidal oscillators in a similar manner.
}

along with the nonlinear device can be considered as a separate first-order nonlinear composite. For example, in the Colpitts oscillator oscillations are actually developed in the active tank resonator. The extra capacitor along with the rest of the bipolar transistor circuit forms a passive first-order nonlinear composite [39]. The Colpitts oscillator can thus be moved to row 8 of the table.

The chaotic behavior observed in a third-order phase-shift oscillator with active nonlinear feedback [40] provides an example of a system with an active zeroth-order nonlinear composite. Hence, it is classified in row 2 of the table. By altering the typical saturation-type characteristic of the device which provides gain in this oscillator such that it becomes piecewise-linear with controllable slopes, chaos can be produced with specific sets of slope values. Analysis of [40] reveals that it is difficult to force the phase-shift oscillator into chaos and our own experience also confirms this fact. Note that a reconstruction of this chaotic oscillator can be carried out by splitting the capacitor which appears in parallel with the active nonlinearity into the sum of two capacitors: one large capacitor and one small (parasitic) capacitor. The small capacitor and the active nonlinearity together form a first-order nonlinear composite. Thus, this chaotic phase-shift oscillator could be moved to row 4 of the table.

In row 3 of the table, two examples are given of systems with an active third-order oscillator and a passive first-order nonlinear composite. The family of chaotic Wien oscillators described in [8] uses a FET-C as the nonlinear composite and a family of second-order Wien-type oscillators. Without considering the dominant pole of the operational amplifier used to synthesize the Wien oscillators, the overall order of the system remains two despite the addition of the FET-C composite. Hence, the active Wien oscillator must be treated as a "weak" thirdorder oscillator. The second example is provided by the FDNRbased chaotic oscillator described in [36]. There, the passive LC tank circuit was activated by placing an FDNR in parallel with it. A portion of the tank's capacitor associated with the switching diode forms the first-order nonlinear composite. 
TABLE I

Classification of Different Autonomous Chaotic Oscillators ASEd on the Oscillator-Nonlinear Composite ARChitectrue (A Stands For ACTIVE AND P STANDS FOR PASSIVE)

\begin{tabular}{|c|c|c|c|c|}
\hline \multicolumn{2}{|c|}{ Oscillator } & \multicolumn{2}{|c|}{ Nonl. Comp. } & \multirow[t]{2}{*}{ Examples } \\
\hline Order & Type & Order & Type & \\
\hline 3 & $\mathrm{~A}$ & 0 & $\mathrm{P}$ & $\begin{array}{l}\text { Chaotic Colpitts oscillator }[3] \text {, Chaotic } \\
\text { Twin-T oscillator [9]. }\end{array}$ \\
\hline 3 & $\mathrm{~A}$ & 0 & $\mathrm{~A}$ & Chaotic phase-shift oscillator [40]. \\
\hline 3 & A & 1 & $\bar{P}$ & $\begin{array}{l}\text { Family of Wien-type oscillators [8], FDNR } \\
\text { chaotic oscillator [36]. }\end{array}$ \\
\hline 3 & $\bar{A}$ & 1 & $\mathrm{~A}$ & $\begin{array}{l}\text { Twin-T-based implementation of Chua's } \\
\text { circuit [19]. }\end{array}$ \\
\hline 3 & $\bar{A}$ & 2 & $\bar{P}$ & $\begin{array}{l}\text { Three-phase oscillator modified using a D- } \\
\text { L composite [12]. }\end{array}$ \\
\hline 2 & $\overline{\mathrm{P}}$ & 1 & $\mathrm{~A}$ & Chua's circuit. \\
\hline 2 & $\mathrm{~A}$ & 1 & $\bar{A}$ & $\begin{array}{l}\text { Rössler's system [18], Saito's double-screw } \\
\text { oscillator [5] and its various realizations } \\
{[6],[20], \text { Chaotic oscillators of }[7],[10],[43]} \\
\text { and [44], Wien-bridge-based implementa- } \\
\text { tions of Chua's circuit [19]. }\end{array}$ \\
\hline 2 & $\bar{A}$ & 1 & $\mathrm{P}$ & $\begin{array}{l}\text { Chaotic oscillator of [4], Chaotic oscilla- } \\
\text { tors based on the FET-C composite [17], } \\
{[32],[45], \text { Based on the FET-L composit }} \\
\text { [11], Multiplier-free Rössler system [21], } \\
\text { Class of hysteresis oscillators [38], Saito's } \\
\text { circuit with a passive nonlinearity [20]. }\end{array}$ \\
\hline 2 & $\overline{\mathrm{A}}$ & 2 & $\mathrm{P}$ & $\begin{array}{l}\text { Circuits based on the D-L composite [10], } \\
{[17],[29] \text {. }}\end{array}$ \\
\hline 2 & $\overline{\mathrm{A}}$ & 2 & $\bar{A}$ & $\begin{array}{l}\text { Quad-Screw oscillator [34], [35], 4D differ- } \\
\text { ential hysteresis oscillator [46]. }\end{array}$ \\
\hline
\end{tabular}

In row 4 of the table, both the oscillator and the nonlinear composite are active and the overall order of the system is four. The Twin- $\mathrm{T}$ based implementation of Chua's circuit is an example of such a construction [19].

Row 5 is dedicated to the case where an active third-order oscillator and a passive second-order nonlinear composite are linked. A circuit with such an architecture is given in [12], where a three-phase oscillator was modified for chaos using the D-L composite; the overall order of the system is five.

Chua's circuit is placed in row 6 with a passive second-order oscillator and a first-order active nonlinear composite formed of the voltage-controlled nonlinear resistor (Chua's diode) and the capacitor in parallel with it. By inspecting the entries of the table, it can be noticed that Chua's circuit is the only entry with a passive oscillator engine. This classification results from the general outlook of the circuit. A closer inspection will reveal that this oscillator is actually active and not passive, as demonstrated in the following section.

In Row 7, a collection of chaotic oscillators that can be shown to have a core second-order sinusoidal oscillator engine is given. This engine is then coupled to an active first-order nonlinear composite. Among this collection are the classical
Rössler system [18], Saito's double-screw hysteresis oscillator [5], [6] and its various realizations [20] (in [20], it was shown that the core of Saito's hysteresis oscillator is a current-controlled $L C-r$ negative resistance oscillator and that it can be replaced with other $R C$ oscillators), the chaotic oscillators of [7], [41]-[44], and the Wien-bridge-based implementations of Chua's circuit [19].

In Row 8, examples of chaotic oscillators with a core secondorder sinusoidal oscillator and a passive first-order nonlinear composite are given. This type includes most circuits based on the FET-C composite [4], [17], [32], [45] (refer also to Sections II-C and III-C of this article) and on the FET-L composite [11]. It also includes the modified Rössler system which uses a switching transistor instead of the artificial multiplier-type nonlinearity [21], a class of hysteresis chaotic oscillators [38], and the single-screw realization which depends on a two-transistor passive differential current-controlled resistor [20].

Chaotic oscillators based on the D-L composite [10], [12], [17], [29] fit in row 9 (refer also to Sections II-B and III-B). Finally, row 10 of the table is dedicated to 4-D oscillators which have two active hysteresis elements and a core second-order oscillator [34], [35], [46]. 


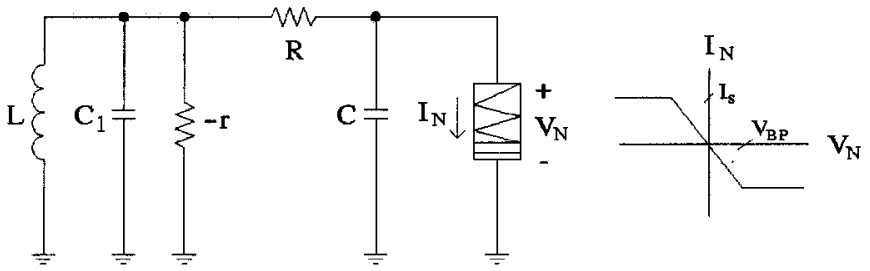

(a)

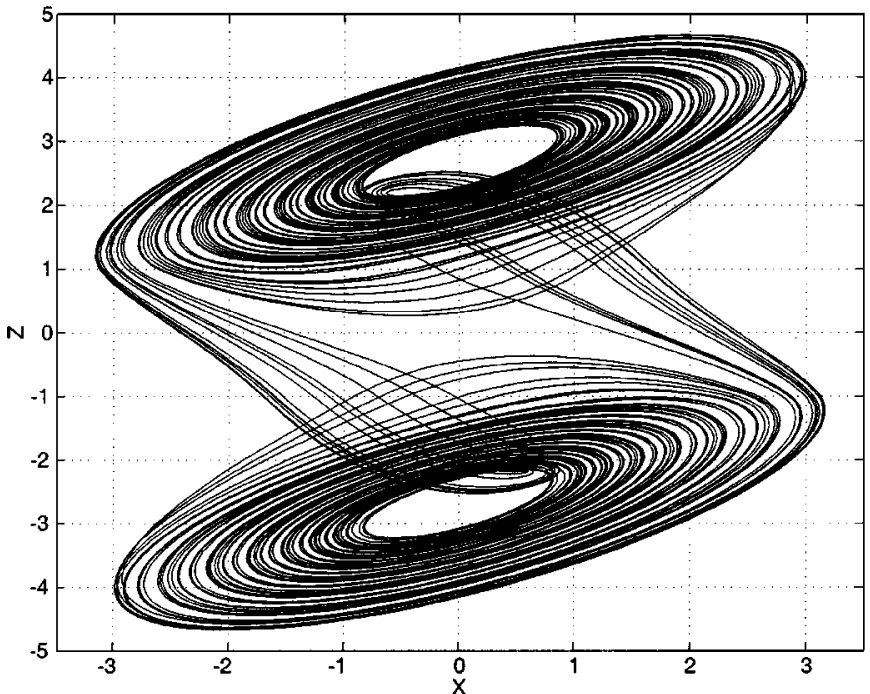

(b)

Fig. 11. (a) Restructured Chua's circuit with a core voltage-controlled $L C-r$ sinusoidal oscillator. (b) Projection of the double-scroll in the $X-Z$ plane $\left(\omega_{r}=2, K=1.5, \Delta=0.01, \varepsilon_{c}=1, A=4\right)$.

\section{B. Reconstruction of Chua's Circuit}

Since the nonlinear characteristics of Chua's diode result from adding in parallel two negative resistors, one of which is linear $(-r)$ while the other is designed to saturate beyond a pair of breakpoints [47], if the linear negative resistor $(-r)$ is detached from the nonlinear resistor and placed in parallel with the passive $L C_{1}$ tank circuit, a classic negative-resistance sinusoidal oscillator results. We show that with this modification, the circuit can preserve the double-scroll dynamics. Thus, we consider the modified Chua's circuit shown in Fig. 11(a) with the simple nonlinear resistor characteristic sketched in the figure. This circuit is described by

$$
\begin{aligned}
C_{1} \dot{V}_{C 1} & =\left(\frac{1}{r}-\frac{1}{R}\right) V_{C 1}+\frac{V_{C}}{R}-I_{L} \\
L \dot{I}_{L} & =V_{C 1}-r_{L} I_{L} \\
C \dot{V}_{C} & =\frac{1}{R}\left(V_{C 1}-V_{C}\right)-I_{N}
\end{aligned}
$$

where $r_{L}$ is a parasitic resistance in series with the inductor. $I_{N}$ is the nonlinear resistor current modeled by

$$
I_{N}= \begin{cases}I_{S} & V_{C} \leq-V_{B P} \\ -\frac{I_{S}}{V_{B P}} V_{C} & -V_{B P}<V_{C}<V_{B P} \\ -I_{S} & V_{C} \geq V_{B P}\end{cases}
$$

where $I_{S}$ is a saturation current and $\pm V_{B P}$ are the voltages at the breakpoints.

By setting $t=\tau / \sqrt{L C_{1}}, X=V_{C 1} / V_{B P}, Y=r I_{L} / V_{B P}$, $Z=V_{C} / V_{B P}, \omega_{r}=r C_{1} / \sqrt{L C_{1}}, K=r / R, \varepsilon_{c}=C / C_{1}$, $\Delta=r_{L} / r$, and $A=r I_{S} / V_{B P}$, the dimensionless form of the above equations becomes

$$
\begin{aligned}
\left(\begin{array}{c}
\omega_{r} \dot{X} \\
\dot{Y} \\
\varepsilon_{c} \omega_{r} \dot{Z}
\end{array}\right)= & \left(\begin{array}{ccc}
1-K & -1 & K \\
\omega_{r} & -\Delta \omega_{r} & 0 \\
K & 0 & -(K+a)
\end{array}\right)\left(\begin{array}{l}
X \\
Y \\
Z
\end{array}\right) \\
& +\left(\begin{array}{c}
0 \\
0 \\
-b
\end{array}\right)
\end{aligned}
$$

and

$$
(a, b)= \begin{cases}(0, A) & Z \leq-1 \\ (-A, 0) & -1<Z<1 \\ (0,-A) & Z \geq 1 .\end{cases}
$$

Indeed, by numerically integrating (21), we still observe the double-scroll attractor, as shown in Fig. 11(b), which represents its $X-Z$ projection for $\omega_{r}=2, K=1.5, \Delta=0.01$, $\varepsilon_{c}=1$, and $A=4$. We note that even with very small values of $\varepsilon_{c}\left(\varepsilon_{c}=0.01\right)$ the double-scroll attractor can still be observed. This means that $C$ can become a parasitic capacitor and that Chua's circuit can then be treated as a slow-fast dynamical system [48]. It is clear that the mechanism by which oscillations are produced in Chua's circuit is via the classical negative-resistance sinusoidal oscillator. The characteristic of the nonlinear resistor sketched in Fig. 11(a) is responsible for switching these oscillations between two parallel planes in the 3-D state-space to form the two scroll surfaces. Smaller values of the switching capacitor result in faster transition between the scrolls.

In the region $-1<Z<1$, the system has its equilibrium point at the origin. It can be shown that for $\varepsilon_{c}=1$ and for $\Delta \rightarrow 0$, the characteristic equation in this region always admits a positive real eigenvalue when the condition $A>K$ is satisfied plus a pair of complex conjugate eigenvalues in the left half-plane when $\omega_{r}^{2}>(1+A) K-A$. Using the values corresponding to Fig. 11(b), the eigenvalues at the origin are $(1.485,-0.252 \pm j 0.89)$. On the scroll surfaces, where $a=0$, the equilibrium points are $(0,-b / K,-b)$ and the calculated eigenvalues at these points are $(-1.067,0.024 \pm j 0.834)$. Note that as oscillations build up on the scroll surfaces, the switching capacitor is being discharged. This discharge process is essential to guarantee that the breakpoint voltages are reached and that switching occurs. During the switching phase, the energy on the oscillator side drops significantly. This manifests itself via the complex eigenvalues with negative real part which appear in this phase. In particular, during the switching phase, the tank circuit is effectively loaded by the equivalent resistor 


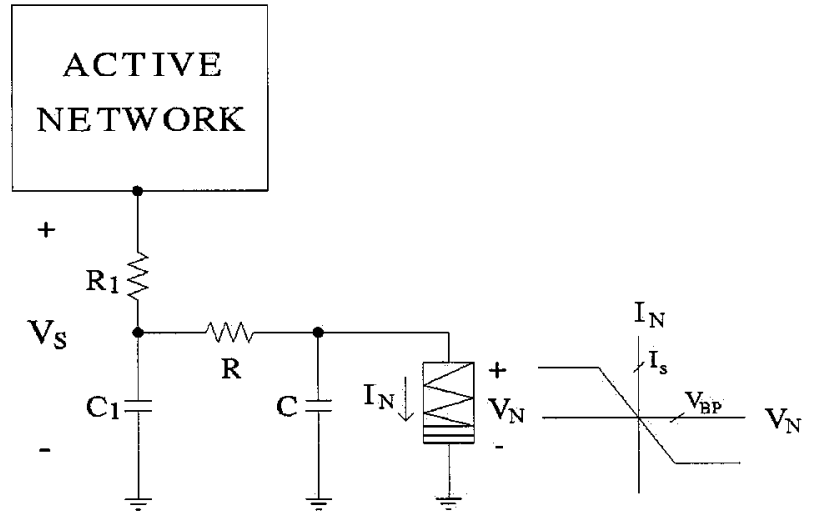

(a)

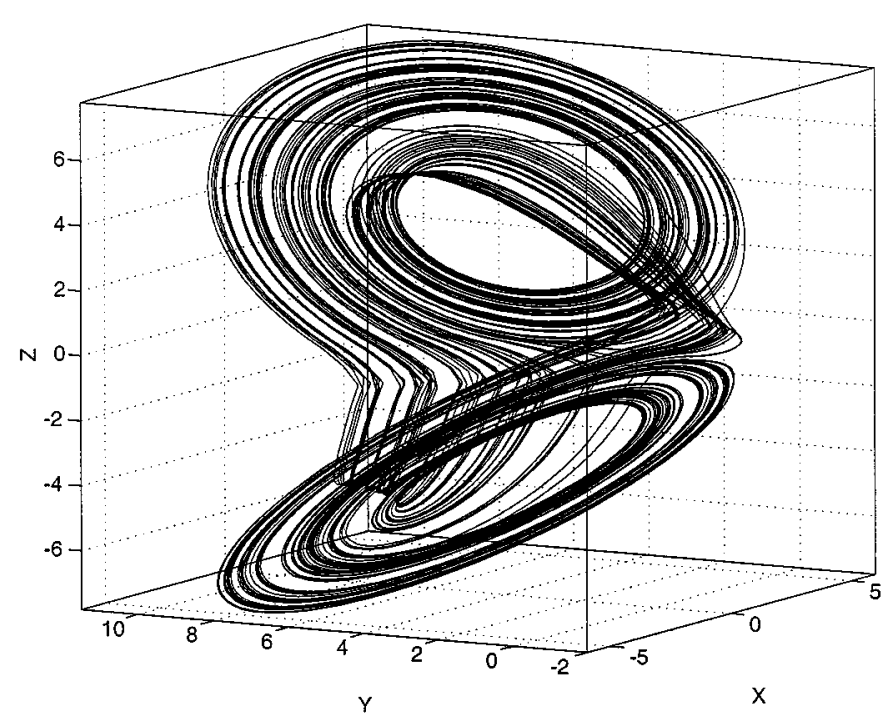

(b)

Fig. 12. (a) Generic $R C$ implementation of Chua's circuit based on the sinusoidal oscillator of Fig. 4(a) when its series $R C$ port is voltage-driven and (b) skewed double-scroll $\left(K_{1}=n=\varepsilon_{r}=\varepsilon_{c}=1, \epsilon=0.55, A=4, K_{2}=1\right.$ if $Z \geq 0$ and $K_{2}=-1$ if $\left.Z<0\right)$.

$R_{e q}=-r R /(R-r)$ which is positive only if $|r|>R$, i.e., $K>1$. This condition coincides with the condition of oscillation of the core sinusoidal oscillator engine in the $X-Y$ plane $(Z=0)$ of the system described by $(21) .{ }^{4}$

It is worth noting that when the linear negative resistor $(-r)$ is detached from the Chua's diode realization of [47], the remaining part will have a nonlinear characteristic slightly different from the simple characteristic modeled by (20). In particular, the outer segments of the characteristic have a positive rather than a zero slope. However, we have chosen to demonstrate that this simple model is sufficient to preserve the doublescroll dynamics.

A Generic RC Realization Of Chua's Circuit: We now consider the configuration shown in Fig. 12(a) where we have replaced the voltage-controlled negative-resistance sinusoidal oscillators in Chua's circuit with the generic $R C$ oscillator of Fig. 4(a). Recall that the sinusoidal oscillator alone is described by (12) when a voltage-driven port is considered.

In addition to the settings used to derive (12), we define $Z=$ $V_{C} / V_{\text {ref }}, \varepsilon_{r}=R_{1} / R$, and $\varepsilon_{c}=C / C_{1}$. Hence for the choice of $V_{S}=K_{1} V_{C 1}-K_{2} V_{C 2}$ and $V_{\text {ref }}=V_{B P}$, Fig. 12(a) is described by [recall that (12) also implies the choice of $C_{1}=C_{2}$; the active network contains $C_{2}$ ]

$$
\begin{aligned}
\left(\begin{array}{c}
\dot{X} \\
\dot{Y} \\
\varepsilon_{c} \dot{Z}
\end{array}\right)= & \left(\begin{array}{ccc}
K_{1}-1-\varepsilon_{r} \pm \epsilon & -K_{2} & \varepsilon_{r} \\
\frac{n+\left(K_{1}-1\right)^{2}}{K_{2}} & 1-K_{1} & 0 \\
\varepsilon_{r} & 0 & -\varepsilon_{r}-a
\end{array}\right) \\
& \cdot\left(\begin{array}{c}
X \\
Y \\
Z
\end{array}\right)+\left(\begin{array}{r}
0 \\
0 \\
-b
\end{array}\right)
\end{aligned}
$$

where $a$ and $b$ are as given by (21b).

\footnotetext{
${ }^{4} \mathrm{By}$ applying the conditions of (2) to (21a) when $Z=0$, it can be shown that the condition for oscillation of the core engine is $K=1+\Delta \omega_{r}^{2}$ and the center frequency of oscillation is $\omega_{0}=\sqrt{1+\Delta(K-1)}$. One can write $K=1+\epsilon$, where $\epsilon$ is the effective control parameter similar to the systems of (7), (9), (13), and (14). For an ideal inductor, $\Delta \rightarrow 0$.
}

We have integrated (22) numerically with $K_{1}=K_{2}=n=$ $\varepsilon_{r}=\varepsilon_{c}=1, \epsilon=0.4$, and $A=4$. The $X-Z$ projection of the observed double-scroll is similar to that shown in Fig. 11(b). Note that the set of parameters $\left(K_{1}, K_{2}, n, \epsilon\right)$ represents only the sinusoidal oscillator engine, while $A$ represents only the nonlinearity. $\varepsilon_{r}$ and $\varepsilon_{c}$ represent the link between the two blocks. In particular, (12) is the limit case of (22) when $\varepsilon_{r} \rightarrow 0$ and $\varepsilon_{c} \rightarrow 0$. We restress that the particular design of the first-order active network [see Fig. 12(a)] is irrelevant so long as it drives the series port with $V_{S}$. Circuit examples of this structure can be found in [19]. Two more generic $R C$ realizations of Chua's circuit are given in [49].

Since changing the sign of $K_{2}$ in (12) changes the direction of rotation in the $X-Y$ plane, we expect to see the same effect in (22). This is a direct result of our ability to isolate the mechanism by which oscillations are produced in the chaotic oscillator. In general, any particular feature which we introduce into the core sinusoidal oscillator engine will automatically be inherited by the chaotic oscillator. Therefore, if an appropriate switching mechanism for $K_{2}$ is used, we expect to obtain a skewed double-scroll. Such a case is demonstrated in Fig. 12(b), where $K_{2}$ is switched to 1 if $Z \geq 0$ and to -1 if $Z<0$.

We conclude our discussion with the following remarks:

1) Our ability to identify the differential equations which represent the sinusoidal oscillator within the derived models of the circuit can be considered as a proof of the validity of Conjecture 1 in this particular case.

2) Chua's circuit now belongs in row 7 of Table I. There, we also find Saito's double-screw oscillator with an identical mechanism for generating oscillations, i.e., via a negative-resistance $L C-r$ oscillator [20]. In fact, Saito's circuit is the dual of Chua's circuit with the negative-resistance oscillator and the nonlinear resistor both current-controlled instead of voltage-controlled. The original circuit proposed by Saito in [5] is of hysteresis nature. However, if the parasitic inductor in series with the 
current-controlled nonlinear resistor (which is essential to correctly model the hysteresis nature of the circuit [50]) is replaced with a physical inductor of value comparable to that of the other inductor (in the $L C-r$ oscillator), the double-screw evolves into a double-scroll. In general, if the parasitic inductor (capacitor) in series (parallel) with the nonmonotone current (voltage)-controlled nonlinear resistor in a hysteresis chaotic oscillator is replaced with a physical inductor (capacitor), the chaotic nature of the circuit can be preserved but the characteristic slow-fast dynamics disappear [38].

A Simple Model with Double-Scroll-Like Dynamics: We propose a simple model which can capture the essential dynamics of double-scroll-like chaotic attractors. The model is given by

$$
\dddot{X}=-a[\ddot{X}+\dot{X}+X-f(X)]
$$

and

$$
f(X)=\operatorname{sgn}(X)=\left\{\begin{aligned}
1, & X \geq 0 \\
-1, & X<0
\end{aligned}\right.
$$

The $\operatorname{sgn}(X)$ nonlinearity is odd-symmetrical and the system has a single parameter $(a)$ via which its dynamical behavior can be changed. The chaotic attractor observed from (23) is shown in Fig. 13(a) for $a=0.8$.

By introducing two new variables, $Y=\dot{X}$ and $Z=\ddot{X}$, it can be shown that the dynamics of (23) in the $Y-Z$ plane $(X=0)$ are given by

$$
\left(\begin{array}{l}
\dot{Y} \\
\dot{Z}
\end{array}\right)=\left(\begin{array}{rr}
0 & 1 \\
-a & -a
\end{array}\right)\left(\begin{array}{l}
Y \\
Z
\end{array}\right)
$$

Thus, the core engine of (23) is a quadrature oscillator in the $Y-Z$ plane with a condition for oscillation given by $a=0$. We can prove the validity of Conjecture 2 in this particular case by considering the two anti-symmetrical halves of the nonlinearity $f(X)$

$$
f(X)^{+}=\left\{\begin{array}{ll}
1 & X \geq 0 \\
0 & X<0
\end{array} \text { and } f(X)^{-}=\left\{\begin{array}{rr}
0, & X \geq 0 \\
-1, & X<0 .
\end{array}\right.\right.
$$

The chaotic attractor of $f(X)$ [see Fig. 13(a)] is a result of merging together the attractors of $f(X)^{+}$and $f(X)^{-}$.

Equation (23) can be synthesized using a integrator-summer architecture, as shown in Fig. 13(b). Here, three lossless noninverting integrators $\left(U_{1}, U_{2}, U_{3}\right)$ are cascaded, followed by an inverting summer stage $\left(U_{4}\right)$. The gain of the inverting summer is equal to the system constant $a$. Thus, by tuning the grounded resistor $a R$ [see Fig. 13(b)], the value of $a$ can be directly changed. The $\operatorname{sgn}(X)$ nonlinearity is realized by an op amp $\left(U_{C}\right)$ operating as a comparator. The switching output voltage of the comparator is routed to the summer after being scaled by $1 / K$, where the value of $K$ is equal to the value of the bias supply of the comparator and is adjusted via resistor $K R$ [see Fig. 13(b)].
An experimental setup of this circuit was constructed ${ }^{5}$ with $R=1 \mathrm{k} \Omega, C=1 \mathrm{nF}, K=9$, and $a=0.8$. All devices were biased with $\pm 9-\mathrm{V}$ supplies. $X-Y$ and $X-Z$ projections of the chaotic attractor are shown in Fig. 13(c) and (d) respectively.

\section{Block Diagram Representation}

Based on our conjectures, we can now draw a block diagram representation of low-order autonomous chaotic oscillators. Fig. 14 shows the block diagram which is composed of an oscillator which has $n$ state variables $\left(X_{O 1} \rightarrow X_{O n}\right)$ and a nonlinear subsystem which has $m$ state variables $\left(X_{S 1} \rightarrow\right.$ $\left.X_{S m}\right)$. One of the state variables might be common between both blocks. In order for this structure to admit chaos, two conditions are necessary.

1) The relation $n+m \geq 3$ must hold. This means that at least three energy storage elements (including parasitic ones) must exist.

2) At least one energy source must exist. This implies that since the oscillator is active, the nonlinear subsystem need not be active as well.

In general, the energy generated in the oscillator is transferred to the nonlinear subsystem. This transfer is controlled by the transfer coefficients $\left(T_{C 0} \rightarrow T_{C n}\right)$ which can be constant coefficients or switching ones that are controlled by the nonlinear subsystem itself. The energy accumulation on the nonlinear subsystem side is sensed by the oscillator via the sensing coefficients $\left(S_{C O} \rightarrow S_{C m}\right)$ which can also be either constants or controlled by the nonlinear subsystem. The feedback around each of the two blocks usually involves one state variable. If the characteristics of the nonlinear subsystem are such that when the accumulated energy reaches a limit value, it is dissipated, and if there exists a set of sensing coefficients to guarantee that the energy on the oscillator side will drop, but not below a minimum limit, as a result of this dissipation, and in addition there exists a set of transfer coefficients to guarantee that energy will reaccumulate in the nonlinear subsystem and reach its limit value, then a mechanism for continuous stretching and folding of the trajectories exists.

Note that if the oscillator is separated from the nonlinear subsystem, it might reach a steady state condition whereby a nonlinear amplitude control mechanism is activated. Therefore, in the chaotic oscillator structure, the transfer and sense coefficients must guarantee that the nonlinear subsystem is activated before the oscillator ever reaches its steady state. In this way, the nonlinear characteristic of the amplitude control mechanism (if it exists) does not contribute to the dynamics of the generated chaos.

From Fig. 14, two important characteristics of this class of chaotic oscillators are apparent.

1) The chaotic signal can be treated as an amplitude modulated signal.

2) The peak power in the frequency spectrum of the generated chaotic signal remains near the oscillator's dominant center frequency. As long as it is possible to extract

\footnotetext{
${ }^{5} \mathrm{AD} 844$ CFOA's were used for $U_{1}, U_{2}, U_{3}$ and $U_{4}$. An AD712 VOA was used for $U_{C}$
} 


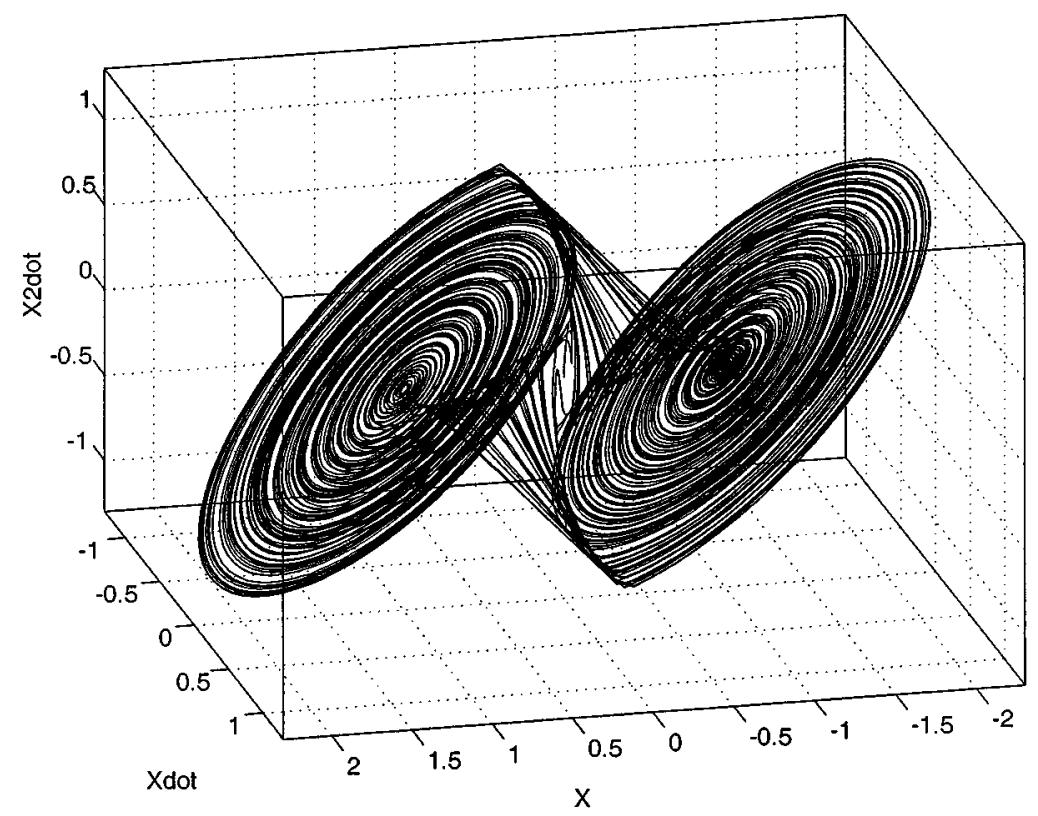

(a)

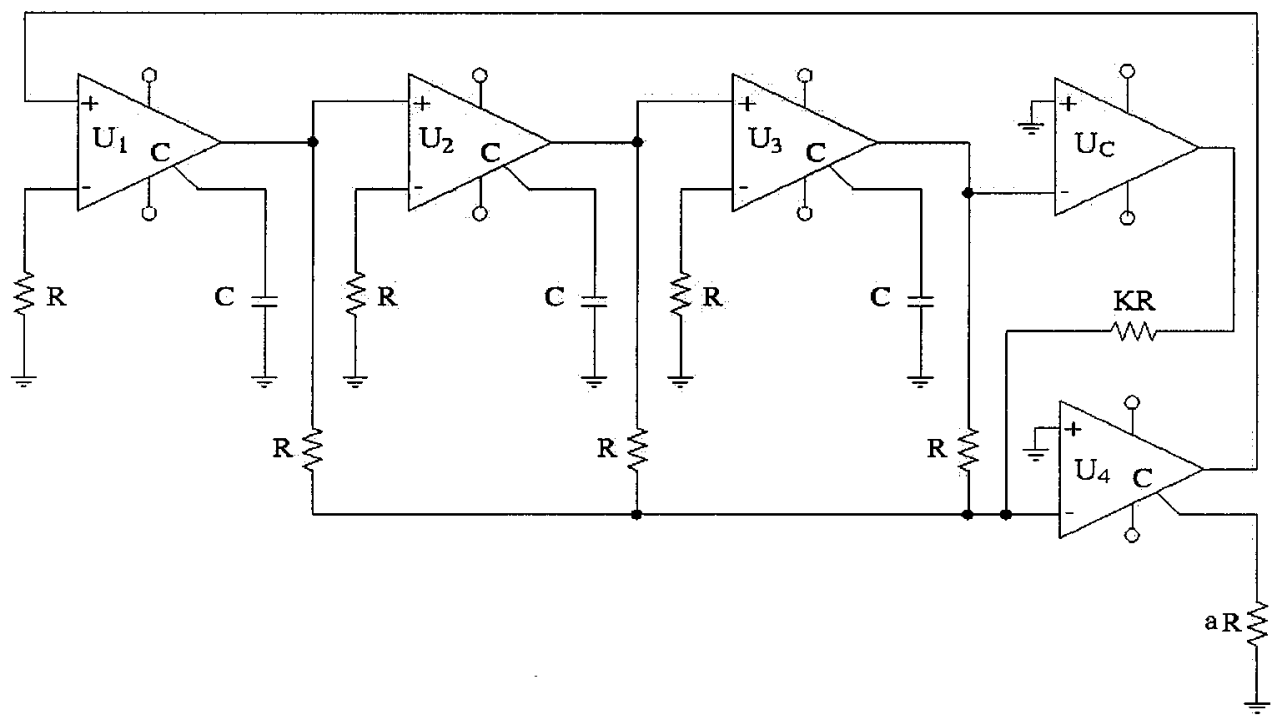

(b)

Fig. 13. (a) The double-scroll-like chaotic attractor observed from (23) with $a=0.8$. (b) A circuit realization based on an integrator-summer architecture. (c)-(d) Experimental observations in the $X-Y$ and $X-Z$ planes ( $X$ axis: $0.5 \mathrm{~V} / \mathrm{div}, Y$ axis: $0.5 \mathrm{~V} / \mathrm{div})$.

the core oscillator engine explicitly form the chaotic oscillator and so long as this engine has a single dominant center frequency, it will be difficult not to recognize this frequency in the chaotic spectrum [19].

\section{Architectures Suitable For Higher Order Chaotic Oscillators}

Two architectures for higher order chaotic oscillators are shown in Fig. 10(b) and (c). In Fig. 10(b), two linear oscillators interact with each other through a nonlinear composite. Exam- ples of this architecture include the first proposed hyperchaotic oscillator [51], where two tank resonators, one of which is active, were coupled via an active nonlinear resistor. Other examples can be found in [52] (two phase-shift oscillators coupled with diodes) as well as some of the circuits of [53]. Recent examples of inductorless hyperchaotic oscillators were proposed in [54] and [55]. Both examples are based on coupling two sinusoidal oscillators via passive diodes.

Other higher order chaotic oscillators follow the structure of Fig. 10(c). In particular, an intermediate filter stage is inserted 


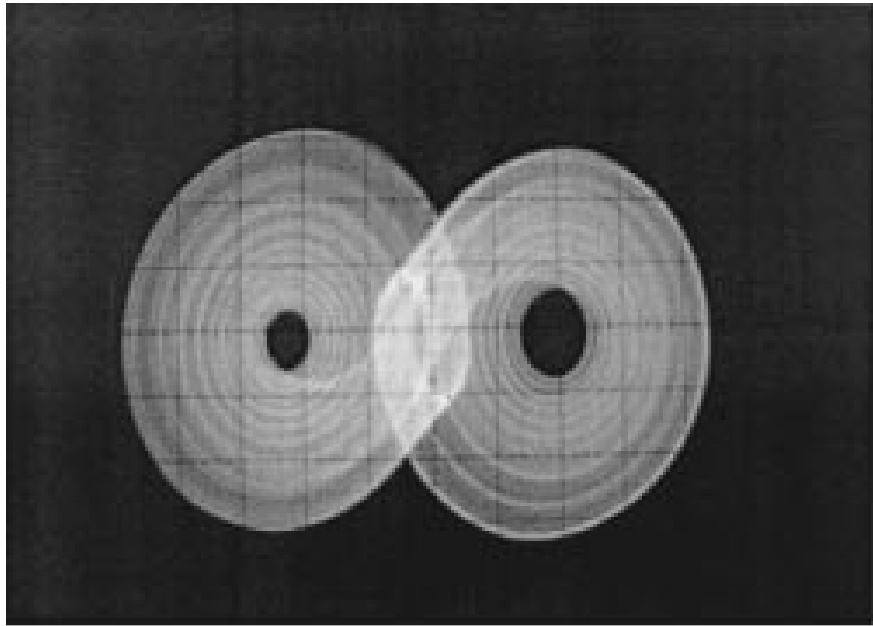

(c)

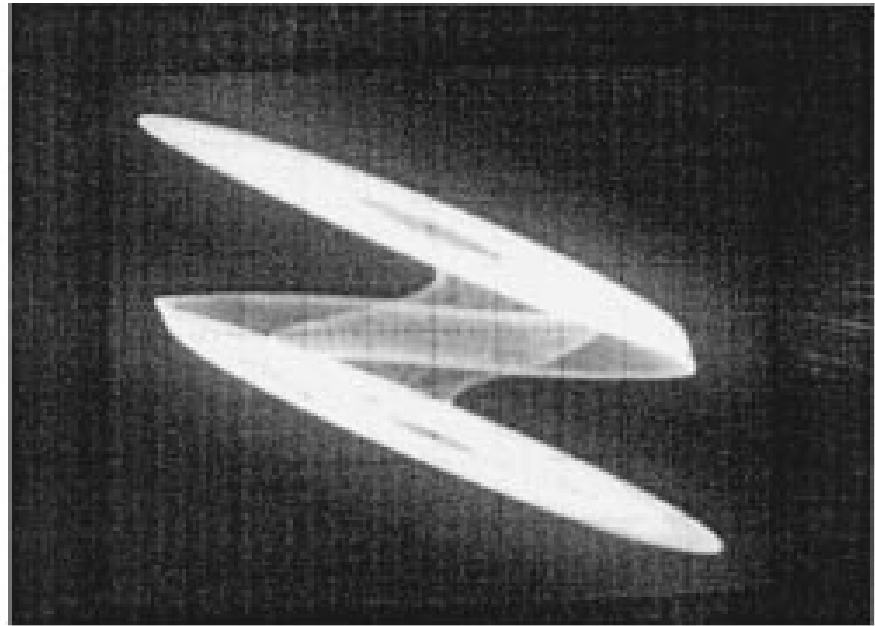

(d)

Fig. 13. (Continued.) (c)-(d) Experimental observations in the $X-Y$ and $X-Z$ planes ( $X$ axis: $0.5 \mathrm{~V} / \mathrm{div}, Y$ axis: $0.5 \mathrm{~V} / \mathrm{div})$.

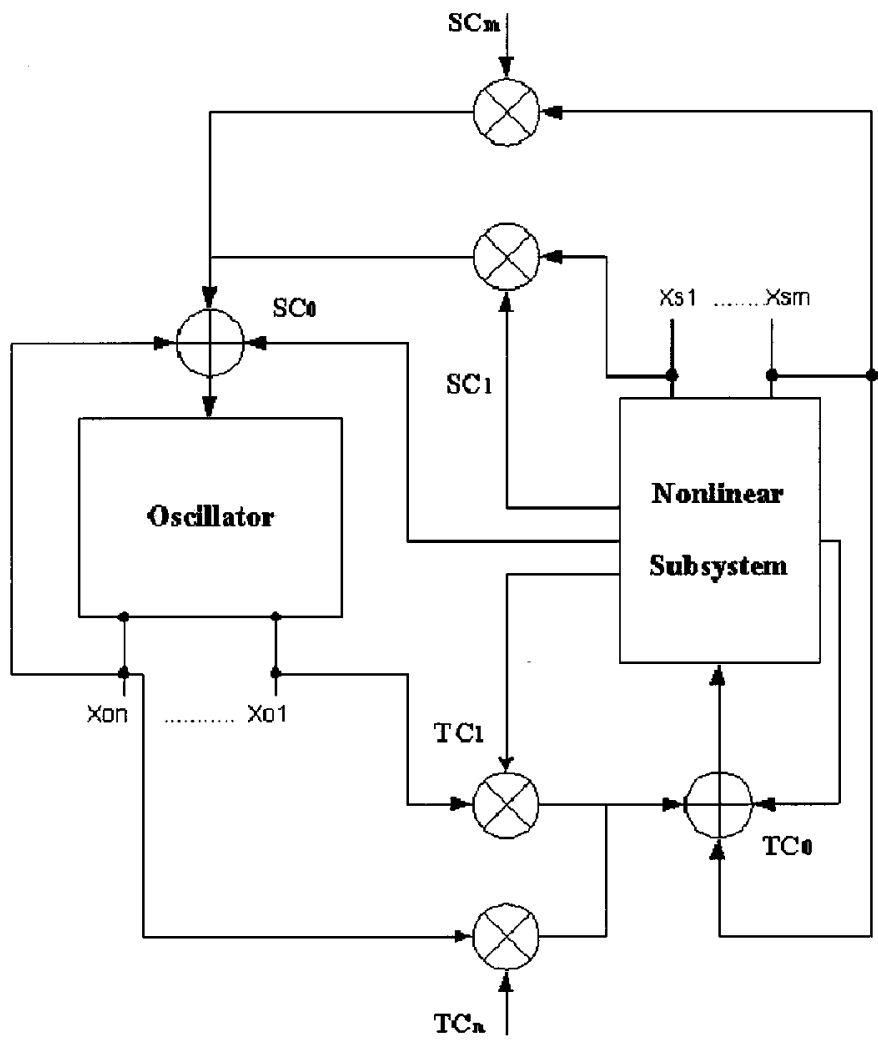

Fig. 14. Block diagram representation of low-dimensional chaotic oscillators based on the oscillator-nonlinear composite structure.

between the oscillator and the nonlinear composite. Examples of such oscillators can be found in [56], where a realization of the Mackey-Glass system was described, and in [57] where a hyperchaotic oscillator was described. Other examples include the circuits of [58] and [59] and the rest of the circuits of [53]. Actually the circuit of [59] is the same as that previously reported by Saito in [57]. In all these examples, the filter (usually a $\pi$ C-L-C section) is purely passive. It was shown in [60] that a single active element is sufficient to generate hyperchaos. However, it is worth noting that increasing the order of the filter by adding extra sections does not imply that the generated chaos expands in a higher dimension [58].

\section{CONCLUSION}

In this work we have described two classes of circuit-independent chaotic oscillators derived from the simplest possible models of two generic second-order $R C$ oscillators. We have shown that the differential equations describing the core oscillator engines can be clearly recognized in the describing equations of the chaotic oscillators. For one of the structures of class II, we have realized that a single parameter of the core oscillator engine can change the phase relation in the $X-Y$ plane without affecting the chaotic dynamics. This observation motivated us to study the well-known Lorenz system and to propose a modified system which is multiplier-free.

We have also restructured Chua's circuit to show that the mechanism by which oscillations are produced in this circuit is via a generic second-order sinusoidal oscillator engine. The coupling of this engine to a first-order active nonlinear composite results in the well-known double-scroll attractor.

A large collection of chaotic oscillators has been classified based on the oscillator-nonlinear composite architecture. We believe that the usual trend of studying the nonlinear dynamics of each chaotic oscillator on individual basis should be replaced by a study of the functional behavior of this architecture. We have stated a conjecture concerning the simplest possible chaotic dynamics that can be observed from this architecture and have proposed a simple equation capturing these dynamics. Chaotic attractors observed in systems with active or hysteresis nonlinearities can be related to this "basic attractor" by considering multiples of it connected together in a manner dictated by the characteristics of the nonlinearity (odd or even-symmetrical), and/or by considering a limit case condition for one or more of its parameters.

\section{REFERENCES}

[1] T. Matsumoto, "A chaotic attractor form Chua's circuit," IEEE Trans. Circuits Syst. I, vol. 31, pp. 1055-1058, 1984. 
[2] G. Q. Zhong and F. Ayrom, "Periodicity and chaos in Chua's circuit," IEEE Trans. Circuits Syst. I, vol. 32, pp. 501-505, 1985.

[3] M. P. Kennedy, "Chaos in the colpitts oscillator," IEEE Trans. Circuits Syst. I, vol. 41, pp. 771-774, 1994.

[4] A. Namajunas and A. Tamasevicius, "Modified Wien-bridge oscillator for chaos," Electron. Lett., vol. 31, pp. 335-336, 1995.

[5] T. Saito and S. Nakagawa, "Chaos from a hysteresis and switched circuit," Phil. Trans. Royal Soc., vol. 353, pp. 47-57, 1995.

[6] S. Nakagawa and T. Saito, "An RC OTA hysteresis chaos generator," IEEE Trans. Circuits Syst. I, vol. 43, pp. 1019-1012, 1996.

[7] A. Namajunas and A. Tamasevicius, "Simple $R C$ chaotic oscillator," Electron. Lett., vol. 32, pp. 945-946, 1996.

[8] A. S. Elwakil and A. M. Soliman, "A family of Wien-type oscillators modified for chaos," Int. J. Circuit Theory Applicat., vol. 25, pp. 561-579, 1997.

[9] - "Two twin-T based op amp oscillators modified for chaos," $J$. Franklin Inst., vol. 335B, pp. 771-787, 1998.

[10] _ , "High-frequency Wien-type chaotic oscillator," Electron. Lett., vol. 34, pp. 1161-1162, 1998.

[11] — - "A family of Colpitts-like chaotic oscillators," J. Franklin Inst., vol. 336, pp. 687-700, 1999.

[12] — , "Three-phase oscillator modified for chaos," Microelectronics J., vol. 30, pp. 863-867, 1999.

[13] T. Matsumoto, L. O. Chua, and G. M. Komura, "The double-scroll," IEEE Trans. Circuits Syst. I, vol. 32, pp. 798-818, 1985.

[14] M. E. Broucke, "One parameter bifurcation diagram for Chua's circuit," IEEE Trans. Circuits Syst. I, vol. 34, pp. 208-209, 1987.

[15] M. P. Kennedy, "On the relationship between the chaotic Colpitts oscillator and Chua's oscillator," IEEE Trans. Circuits Syst. I, vol. 42, pp. 376-379, 1995.

[16] A. S. Elwakil and M. P. Kennedy, "Toward a methodology for designing autonomous chaotic oscillators," in Proc. 6th Int. Workshop Nonlinear Dynamics of Electronic Systems NDES'98, Budapest, 1998, pp. 79-82.

[17] — "A semi-systematic procedure for producing chaos from sinusoidal oscillators using diode-inductor and FET-capacitor composites," IEEE Trans. Circuits Syst. I, vol. 47, pp. 582-590, 2000.

[18] O. E. Rössler, "An equation for continuous chaos," Phys. Lett. A, vol. 57, pp. 397-398, 1976.

[19] A. S. Elwakil and M. P. Kennedy, "Chua's circuit decomposition: A systematic design approach for chaotic oscillators," J. Franklin Inst., vol. 337, pp. 251-265, 2000.

[20] - "Chaotic oscillators derived from Saito's double-screw hysteresis oscillator," IEICE Trans. Fundamentals, vol. 82, pp. 1769-1775, 1999.

[21] — "Chaotic oscillator configuration using a frequency dependent negative resistor," J. Circuits Syst. Computers, vol. 9, pp. 229-242, 1999.

[22] E. N. Lorenz, "Deterministic non periodic flow," J. Atmos. Sci., vol. 20, pp. 130-141, 1963.

[23] N. Boutin, "Two new single op amp $R C$ bridge-T oscillator circuits," Proc. IEE Circuits Devices Syst., vol. 130, pp. 222-224, 1983.

[24] A. M. Soliman, "Novel oscillators using current and voltage followers," J. Franklin Inst., vol. 335, pp. 997-1007, 1998.

[25] — - "Current mode CCII oscillators using grounded capacitors and resistors," Int. J. Circuit Theory Applicat., vol. 26, pp. 431-438, 1999.

[26] P. A. Martinez, J. Sabdell, C. Aldea, and S. Celma, "Variable frequency sinusoidal oscillators based on CCII+," IEEE Trans. Circuits Syst. I, vol. 46, pp. 1386-1390, 1999

[27] L. O. Chua and P. M. Lin, Computer-Aided Analysis of Electronic Circuits. Englewood Cliffs, NJ: Prentice-Hall, 1975.

[28] L. O. Chua, C. A. Desoer, and E. S. Kuh, Linear and Nonlinear Circuits. New York: McGraw-Hill, 1987.

[29] A. S. Elwakil and M. P. Kennedy, "Novel chaotic oscillator configuration using a diode-inductor composite," Int. J. Electron., vol. 87, pp. 397-406, 2000.

[30] R. Tokunaga, L. Chua, and T. Matsumoto, "Bifurcation analysis of a cusp-constrained piecewise-linear circuit," Int. J. Circuit Theory Applicat., vol. 17, pp. 283-346, 1989.

[31] A. S. Elwakil and A. M. Soliman, "Two modified for chaos negative impedance converter op amp oscillators with symmetrical and antisymmetrical nonlinearities," Int. J. Bifurcation Chaos, vol. 8, pp. 1335-1346, 1998.

[32] - "Current conveyor chaos generators," IEEE Trans. Circuits Syst. I, vol. 46, pp. 393-398, 1999.
[33] R. Gilmore, "Topological analysis of chaotic dynamical systems," Rev. Modern Phys., vol. 70, pp. 1455-1529, 1998.

[34] M. Kataoka and T. Saito, "Design of 4-D chaotic oscillators consisting of 2-port VCCS's and capacitors," in Proc. 7th Int. Specialist Workshop Nonlinear Dynamics Electron. Syst. NDES'99, Bornholm, 1999, pp. 197-200.

[35] — - "A 4-D chaotic oscillator with a hysteresis 2-port VCCS: The first example of chaotic oscillators consisting of 2-port VCCS's and capacitors," in Proc. IEEE Symp. Circuits Syst. ISCAS'99, Orlando, 1999, pp. $418-421$.

[36] A. S. Elwakil and M. P. Kennedy, "Chaotic oscillator configuration using a frequency dependent negative resistor," Int. J. Circuit Theory Applicat., vol. 28, pp. 69-76, 2000.

[37] L. O. Chua, J. Yu, and Y. Yu, "Negative resistance devices," Int. J. Circuit Theory Applicat., vol. 11, pp. 161-185, 1983.

[38] A. S. Elwakil and M. P. Kennedy, "Systematic realization of a class of hysteresis chaotic oscillators," Int. J. Circuit Theory Applicat., vol. 28, pp. 319-334, 2000

[39] G. Sarafian and B. Z. Kaplan, "Is the colpitts oscillator a relative of Chua's circuit,” IEEE Trans. Circuits Syst. I, vol. 42, pp. 373-376, 1995.

[40] M. J. Ogorzalek, "Order and chaos in a third-order RC ladder network with nonlinear feedback," IEEE Trans. Circuits Syst. I, vol. 36, pp. 1221-1230, 1989.

[41] A. Tamasevicius, "Reproducible analogue circuit for chaotic synchronization," Electron. Lett., vol. 33, pp. 1105-1106, 1997.

[42] M. Storace and M. Parodi, "Simple realization of hysteresis chaos generator," Electron. Lett., vol. 34, pp. 10-11, 1998.

[43] T. Saito and K. Mitsubori, "Chaos due to impulsive switching," in Proc. European Conf. Circuit Theory and Design ECCTD'97, Budapest, 1997, pp. 1019-1024.

[44] T. Saito, "A chaos generator based on a quasiharmonic oscillator," IEEE Trans. Circuits Syst. I, vol. 32, pp. 320-331, 1985.

[45] A. S. Elwakil and A. M. Soliman, "Current mode chaos generator," Electron. Lett., vol. 33, pp. 1661-1662, 1997.

[46] J. E. Varrientos and E. Sanchez-Sinencio, "A 4D chaotic oscillator based on a differential hysteresis comparator," IEEE Trans. Circuits Syst. I, vol. 45, pp. 1-10, 1998.

[47] M. P. Kennedy, "Robust op amp realization of Chua's circuit," Frequenz, vol. 46, pp. 66-80, 1992.

[48] B. Rossetto, "Chua's circuit as a slow-fast autonomous dynamical system," J. Circuits Syst. Computers, vol. 3, pp. 483-496, 1993.

[49] A. S. Elwakil and M. P. Kennedy, "Generic RC realizations of Chua's circuit," Int. J. Bifurcation Chaos, vol. 10, pp. 1981-1985, 2000.

[50] M. P. Kennedy and L. O. Chua, "Hysteresis in electronic circuits: A circuit theorist's perspective," Int. J. Circuit Theory Applicat., vol. 19, pp. 471-515, 1991.

[51] T. Matsumoto, L. O. Chua, and K. Kobayashi, "Hyperchaos laboratory experiment and numerical confirmation," IEEE Trans. Circuits Syst. I, vol. 33, pp. 1143-1147, 1986.

[52] Y. Hosokawa, Y. Nishio, and A. Ushida, " $R C$ transistor chaotic circuit using phase-shift oscillators," in Proc. Int. Symp. Nonlinear Theory its Applicat. NOLTA'98, Switzerland, 1998, pp. 603-606.

[53] A. Tamasevicius, "Hyperchaotic circuits: state of the art," in Proc. 5th Int. Specialist Workshop Nonlinear Dynamics Electron. Syst. NDES'97, Moscow, 1997, pp. 97-102.

[54] A. Tamasevicius, A. Cenys, G. Mykolaitis, A. Namajunas, and E. Lindberg, "Hyperchaotic oscillator with gyrators," Electron. Lett., vol. 37, pp. 542-544, 1997.

[55] A. S. Elwakil and M. P. Kennedy, "Inductorless hyperchaos generator," Microelectronics J., vol. 30, pp. 739-743, 1999.

[56] A. Namajunas, K. Pyragas, and A. Tamasevicius, "An electronic analog of the Mackey-Glass system," Phys. Lett. A, vol. 201, pp. 43-46, 1995.

[57] T. Saito, "A simple hyperchaos generator including one ideal diode," IEICE Trans. Fund., vol. E75, pp. 294-298, 1992.

[58] A. Tamasevicius, G. Mykolaitis, and A. Cenys, "Chaos diode," IEE Proc. Circuits Devices Syst., vol. 145, pp. 361-362, 1998.

[59] A. Tamasevicius, A. Namajunas, and A. Cenys, "Simple 4D chaotic oscillator," Electron. Lett., vol. 32, pp. 957-958, 1996.

[60] A. Tamasevicius and A. Cenys, "Hyperchaos in dynamical systems with a monoactive degree of freedom," Chaos Solitons Fractals, vol. 9, pp. 115-119, 1998. 


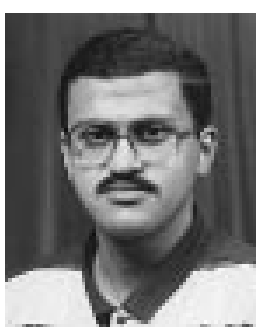

Ahmed S. Elwakil (M’99) was born in Cairo, Egypt. $\mathrm{He}$ received the B.Sc. and M.Sc. degrees from the Department of Electronics and Communications at Cairo University and the Ph.D. degree from the Department of Electronic and Electrical Engineering at the National University of Ireland, Dublin.

He has been a Staff Member at the Egyptian Nuclear Research Center since 1996. His research interests primarily include the areas of analog circuit design, nonlinear dynamics, and chaos generation. He is author and co-author of more than 50 articles in

these areas.

Dr. Elwakil is a member of the IEEE Technical Committee on Nonlinear Circuits and has served as a reviewer for many journals and conferences. $\mathrm{He}$ has served as an instructor for two basic VLSI design courses organized by the United Nations University and is a regular associate of the International Center For Theoretical Physics (ICTP). He is also a Senior Member of the Institute of Electrical Engineers (IEE).

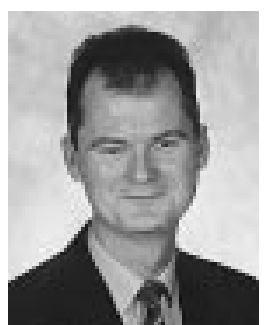

Michael Peter Kennedy (''84-M'91-F'98) received the B.E. degree in electronics from the National University of Ireland in 1984 and the M.S. and Ph.D. degrees from the University of California at Berkeley (UC Berkeley) in 1987 and 1991, respectively, for his contributions to the study of neural networks and nonlinear dynamics.

He worked as a Design Engineer with Philips Electronics, a Postdoctoral Research Engineer with the Electronics Research Laboratory, UC Berkeley, and as a Visiting Professor with the EPFL, Switzerland. He returned to University College Dublin (UCD) in 1992 as a College Lecturer in the Department of Electronic and Electrical Engineering, where he taught electronic circuits, computer-aided circuit analysis, and nonlinear circuits and systems, and directed the undergraduate electronics laboratory. $\mathrm{He}$ was appointed Statutory Lecturer at UCD in 1996 and Associate Professor in 1999. In 1999, he was appointed Professor of Microelectronics and Head of the Department of Microelectronic Engineering at University College Cork. He has published more than 180 articles in the area of nonlinear circuits and systems and has taught courses on nonlinear dynamics and chaos in England, Switzerland, Italy, and Hungary. He has held visiting research positions at the EPFL, AGH Krakow, TU Budapest, and UC Berkeley and has acted as a consultant for microelectronics design companies, the European Commission, and UC Berkeley. His research interests include the simulation, design, and analysis of nonlinear dynamical systems for applications in communications and signal processing. He is also engaged in research into algorithms for mixed-signal testing.

Dr. Kennedy received the 1991 Best Paper Award from the International Journal of Circuit Theory and Applications and the Best Paper Award at the European Conference on Circuit Theory and Design 1999. He serves as a reviewer for a number of conferences, journals and publishers, as well as national science funding agencies in Europe, Asia, and the United States. He served as an Associate Editor of the IEEE TRANSACTIONS ON CIRCUITS AND SYSTEMS-I from 1993 to 1995, as Guest Editor of Special Issues on "Chaos Synchronization and Control" (1997), "Advances in Nonlinear Electronic Circuits" (1999) and "Noncoherent Chaotic Communications" (2000). He was Chair of the IEEE Technical Committee on Nonlinear Circuits and Systems during 199899 and is currently Associate Editor of the IEEE TRANSACTIONS ON CIRCUITS AND SYSTEMS-II. He is the founding Chairman of the Ireland Chapter of the IEEE Solid-State Circuits Society and Chairman of the IEE Circuits and Systems Professional Group. In 2000, he was awarded the Millenium Medal of the IEEE Circuits and Systems Society. 